Boletín de la Sociedad Geológica Mexicana

VOLUMEN 67, NÚM. 2, 2015, P. 255-272

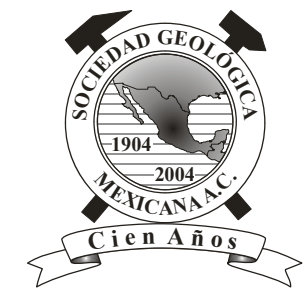

\title{
Influencia de los paleosuelos en los procesos exógenos modernos en la porción noreste de la Cuenca de México
}

\author{
Elizabeth Solleiro-Rebolledo ${ }^{1,2, *}$, Sergey Sedov ${ }^{1}$, Svetlana Sycheva ${ }^{3}$, Serafín Sánchez Pérez ${ }^{4,5}$, \\ Konstantin Pustovoitov ${ }^{6,7}$, Daniela Sauer ${ }^{6,8}$ \\ ${ }^{1}$ Instituto de Geología, Universidad Nacional Autónoma de México, Ciudad Universitaria, C.P. 04510, México D.F. \\ ${ }^{2}$ Institute of Geography and Geology, University of Würzburg. Würzburg, 97074, Germany. \\ ${ }^{3}$ Institute of Geography, Russian Academy of Sciences. Moscow, Russia. \\ ${ }^{4}$ Posgrado en Ciencias de la Tierra, Instituto de Geología, Universidad Nacional Autónoma de México. \\ ${ }^{5}$ Escuela Nacional de Antropología e Historia, México, D.F. \\ ${ }^{6}$ Institute of Soil Science and Land Evaluation, University of Hohenheim, Germany. \\ ${ }^{7}$ Institute of Near Eastern Archaeology, University of Freiburg, Germany. \\ ${ }^{8}$ Institute of Geography, University of Göttingen, Germany. \\ *solleiro@geologia.unam.mx
}

\begin{abstract}
Resumen
Dada la importancia que revisten los suelos en los procesos que ocurren en la zona crítica (zona de interacción entre agua, suelo, aire, organismos), en este trabajo presentamos la distribución temporal y espacial de una secuencia de paleosuelos arcillosos con carbonatos secundarios (capas de caliches y carbonatos diseminados) en el sector nororiental de la Cuenca de México, particularmente en el valle de Teotihuacán. El propósito de este trabajo consiste en reconstruir la distribución temporal y espacial de la cubierta de suelos del pasado para entender su relación con la estructura actual de suelos y sedimentos en este sector semiárido de la cuenca de México. Con esta información, determinar la participación de estos materiales en la Zona Crítica (ZC). Para ello, se ha llevado a cabo un transecto de dirección norte-sur, que inicia en las elevaciones del Cerro Gordo, cruza el valle de Teotihuacán y termina en la Sierra Patlachique, describiendo perfiles de suelo en diferentes posiciones geomorfológicas. El marco cronológico se ha establecido con base en fechamientos de radiocarbono realizados en las capas de caliches. De acuerdo a la cronología, al tipo y grado de pedogénesis, y a los procesos de sedimentación, se han reconocido cuatro unidades. La primera unidad, denominada Paleosuelos del Pleistoceno, fase I (50000 - 20000 años AP), está compuesta de paleosuelos arcillosos, los cuales son Luvisoles crómicos y estágnicos, dependiendo de su posición en el paisaje. Estos paleosuelos muestran tanto carbonatos secundarios diseminados y en fracturas, como capas de caliches. En la segunda unidad, Paleosuelos del Pleistoceno, fase II (20000 -10000 años AP), dominan los sedimentos sobre los suelos; únicamente en el fondo del Valle, se han encontrado Gleysoles cortados por canales aluviales. La unidad, Paleosuelos del Holoceno, fase I (10000 a 1000 años AP) se caracteriza por presentar suelos poligenéticos (Vertisoles), fuertemente modificados por los procesos antrópicos, durante las diversas fases de ocupación pre-hispánica. La última unidad, Suelos del Holoceno, fase II, abarca el último milenio, en donde se destacan procesos de erosión e inestabilidad del paisaje. La tendencia principal en la distribución espacial de los paleosuelos es la siguiente: las unidades antiguas (Luvisoles, capas de caliches y Vertisoles) están sepultadas profundamente en el fondo del valle. Sin embargo, en los taludes y elevaciones montañosas, pueden aparecer cerca o directamente sobre la superficie, siendo incorporados en el manto de suelos actual. Las interrelaciones entre los diferentes paleosuelos a lo largo del transecto estudiado, junto con las observaciones micromorfológicas, apoyan la hipótesis de una relación evolutiva entre los Luvisoles pleistocénicos y los Vertisoles del Holoceno. Consideramos que estas diferentes unidades repercuten en los procesos hidrológicos en el valle y que las capas de caliche formadas en los ambientes pleistocénicos pueden afectar la geoquímica del agua subterránea así como sus firmas isotópicas.
\end{abstract}

Palabras clave: paleosuelos, Teotihuacán, caliches, Luvisoles, Vertisoles, cuenca de México 


\begin{abstract}
As soils are important elements for the processes involved in the critical zone (the zone of interaction between water, soil, air, and organisms), in this work we present the temporal and spatial distribution of a clayey-paleosol sequence with secondary carbonates (layers of caliche and disseminated carbonates) in the northeast sector of the Mexico Basin, particularly in the Teotihuacan Valley. The goal of this paper is the temporal and spatial reconstruction of the past soil cover to understand its relationship with the present day soils and sediments in this semiarid sector of the Mexico Basin. With this information the involvement of these materials in the critical zone could be determined. To comply with these objectives, a north-south transect was carried out, starting in the Cerro Gordo, crossing the Teotihuacan valley and ending in the Sierra Patlachique, describing soil profiles located in different geomorphological positions. The chronological framework has been established with radiocarbon dates of the caliche layers. According to this chronology, the type and degree of pedogenesis, as well as the sedimentation processes, four units have been recognized. The first unit is named the Pleistocene Paleosols, phase I (50000 - 20000 years BP), and is composed of clayey paleosols, chromic and stagnic Luvisols, depending on their position in the landscape. These paleosols contain pedogenic carbonates, disseminated and in fractures, and caliche layers. In the second unit, the Pleistocene Paleosols, phase II (20000-10000 years BP), sediments predominate over soils; only in the valley floor some Gleysols are found to be cut by alluvial channels. The unit, Paleosols of the Holocene Paleosols unit, phase I (10000 to 1000 years BP), is characterized by polygenetic soils (Vertisols) strongly modified by anthropic processes during distinct phases of pre-hispanic occupation. The last unit, the Holocene Soils, phase II, covers the last millennium when erosional processes and unstable landscape dominate. The paleosols (Luvisols, caliche layers, and Vertisols) are deeply buried at the bottom of the valley. However, on the slopes and hilltop positions, they can appear close to or directly on the surface, being incorporated in the present day soil mantle. Spatial interrelations of different paleosols along the studied transect together with micromorphological observations support the hypothesis of an evolutionary link between the Pleistocene Luvisols and the Holocene Vertisols. We propose that these different units are involved in the hydrological processes in the valley and that the caliche layers, formed in the Pleistocene environments, can affect the geochemistry of the groundwater as well as its isotopic signatures.
\end{abstract}

Keywords: paleosols, Teotihuacan, caliches, Luvisols, Vertisols, Basin of Mexico

\section{Introducción}

La Zona Crítica (ZC) se considera como el medio de interacción entre la roca, el suelo, el agua, el aire y los organismos vivos (National Research Council, 2001). El suelo es la conexión principal de la ZC y representa una geomembrana a través de la cual las sustancias (incluida el agua) migran, participando activamente en el intercambio de energía, gases y sólidos, entre las otras esferas (litosfera, atmosfera, biosfera, hidrosfera) (Lin, 2010). De hecho, las relaciones entre el suelo y el agua han sido replanteadas en los últimos años, emergiendo una nueva disciplina, la hidropedología, con la pretensión de establecer el papel que juegan los suelos en los procesos hidrológicos, con énfasis en su posición en el paisaje (Lin et al., 2006).

Sin embargo, en los estudios hidropedológicos, se tiene que tomar en cuenta no solo el tipo y extensión de la cubierta edáfica superficial, sino la presencia de suelos sepultados o paleosuelos. Estos objetos, formados en superficies del pasado, representan otro factor que influye activamente en los flujos dentro de la ZC, ya que sus propiedades pueden diferir de las encontradas en los suelos modernos y, por lo tanto, repercutir en la composición del agua subterránea.

\subsection{Los suelos de la Cuenca de México}

Como es sabido, la cuenca de México es de tipo endorreico, cerrada por el vulcanismo de la Sierra de Chichinautzin (Mooser, 1975). Esta región, que albergó un complejo sistema lacustre y que fue afectada por el vulcanismo y la actividad tectónica del Cuaternario, no parece ser el lugar ideal para el estudio de suelos, ya que durante la mayor parte de su historia permaneció bajo agua. En consecuencia, los materiales que la componen son principalmente, de origen lacustre y volcánico. A inicios del siglo XVII, se construyó el tajo de Nochistongo, con el objetivo de drenar la cuenca y evitar las inundaciones que afectaban regularmente a la Ciudad de México. Sin embargo la reducción final del lago no ocurrió sino hasta el siglo XX. Por esta razón, los suelos de la zona son muy jóvenes y han sido fuertemente modificados por el crecimiento urbano. Por lo tanto, para conocer las características de los suelos de la zona, es necesario buscar sitios en donde los factores pedogenéticos hayan actuado en los sedimentos por un tiempo suficiente para formarlos. Estos sitios representan las áreas drenadas, como son los límites del lago y las superficies de mayor elevación. Sin embargo, las zonas montañosas como la Sierra de las Cruces, al occidente, o la 
Sierra de Guadalupe al norte (Figura 1), no muestran suelos con alto grado de desarrollo (Flores-Román et al., 2009), debido a las pendientes pronunciadas que promueven los procesos de remoción en masa (García-Palomo et al., 2006; Carlos-Valerio, 2010).

Por el contrario, la zona centro-nororiental de la Cuenca, en donde se localiza el valle de Teotihuacán, representa un área en donde la evolución geo-pedológica ha permitido el desarrollo de suelos y paleosuelos. En este valle, se observan ciclos de estabilidad e inestabilidad ambiental que repercuten en los procesos pedogenéticos y de erosión/ sedimentación (Solleiro-Rebolledo et al., 2011). Durante las fases inestables, los procesos geomórficos originan el movimiento de sedimentos desde las posiciones de mayor elevación y su sedimentación en las partes bajas del valle. En las fases de estabilidad, estos procesos cesan, de tal manera que los factores de formación de suelo actúan en el desarrollo de la cubierta edáfica.

De acuerdo con las investigaciones desarrolladas durante los últimos años en Teotihuacán, se ha podido reconocer la presencia de paleosuelos con diversas propiedades y fases de desarrollo, que representan diferentes momentos de la historia geológico-ambiental (Solleiro-Rebolledo et al., 2006, 2011; Sedov et al., 2010; Sánchez et al., 2013). Estos paleosuelos han demostrado cambios drásticos en los ambientes del Cuaternario tardío como consecuencia de modificaciones en los factores climáticos y bióticos, entre los que se incluye la acción del hombre.

Los paleosuelos del valle de Teotihuacán se encuentran sepultados o en superficie y, a pesar de que representan cuerpos relictos de ambientes del pasado, juegan un papel importante en las condiciones actuales del valle. Cabe señalar que los estudios paleopedológicos anteriores, realizados en esta zona, comprenden un análisis detallado, pero limitado a algunos perfiles selectos. Por lo tanto, la distribución e interrelación espacial de los componentes de la cubierta edáfica (incluyendo sus elementos relictos) son poco conocidos. Por lo anterior, el propósito de este trabajo radica en reconstruir la distribución temporal y espacial de la cubierta de suelos actuales y pasados para establecer su relación en la estructura actual de suelos y sedimentos en el sector semiárido de la cuenca de México. Con esta información, determinar la participación de estos materiales en la ZC y en la recarga del acuífero.

\subsection{Impacto humano en Teotihuacán}

La presencia humana en la porción norte de la Cuenca de México se ha registrado desde hace 11000 años (González et al., 2003), de acuerdo con la información procedente de varias localidades. En ellas se han recuperado huesos y cráneos humanos, en sitios como Tlapacoya y El Peñón. De esta manera, puede decirse que la influencia humana en los ambientes del Holoceno ha sido prácticamente constante, intensificándose en los últimos 3000 años, durante los cuales, tanto la agricultura y como el desarrollo urbano, han modificado sustancialmente los paisajes.

Los primeros pobladores se dedicaban a actividades como la caza, la pesca y la recolección, gracias a los hábitats desarrollados en el Holoceno temprano (González et al., 2006). Sin embargo, la información sobre este primer periodo de ocupación en la zona de Teotihuacán se encuentra fragmentada (McClung de Tapia, 2012). Por el contrario, los últimos tres a cinco milenios de poblamiento, se han estudiado de una manera intensiva, desde diversas perspectivas (e.g. Millon, 1973; Sanders et al., 1979; McClung de Tapia, 1987; Manzanilla, 1997).

La actividad agrícola en el valle inicia en el año 1500 a.C. (Sanders et al., 1979), pero es a partir del año 200 d.C., que se registra un crecimiento demográfico importante en Teotihuacán, como consecuencia de los flujos migratorios procedentes del centro del país y, sobre todo, del sur de la Cuenca de México, por la intensa actividad volcánica (McClung de Tapia, 2012). De hecho, la ciudad alcanza una población de 125000 habitantes entre los años 350 y 550 d.C. (Millon, 1973). Estos pobladores efectuaron modificaciones importantes en el paisaje debido a la utilización de sistemas agrícolas que incluyeron el desarrollo de sistemas de irrigación, complejos hidráulicos y terrazas de cultivo (Cabrera, 2005). Los suelos de la región muestran evidencias de estas prácticas agrícolas, entre las que destacan fragmentos de carbón, agrocutanes, restos de polen y fitolitos de maíz, concentraciones elevadas de fósforo y rasgos reductomórficos relacionados con el riego (RiveraUria et al., 2007; Sedov et al., 2009; Sánchez et al., 2013).

Asimismo, se ha documentado el deterioro del paisaje, por la construcción del centro ceremonial de Teotihuacán, el cual implicó la remoción de grandes cantidades de materiales, como árboles, rocas, sedimentos y suelos agrícolas, estos últimos usados en el interior de las pirámides (Barba, 2005; Rivera-Uria et al., 2007; Sánchez et al., 2013).

\section{Características ambientales del valle de Teotihuacán}

El Valle de Teotihuacán se ubica en la porción NE de la Cuenca de México (Figura 1). En su límite norte se tienen varios edificios volcánicos, formados durante el Plioceno y el Pleistoceno (Vázquez y Jaimes, 1989), como Malinalco, Cerro Gordo, Cerro Soledad y Texuaca; al sur y sureste se encuentra la Sierra Patlachique, al oeste el Cerro Chiconauhtla y al este la Sierra de Soltepec. El Cerro Gordo, un volcán cuaternario de composición basáltica, representa la posición más elevada (3050 msnm). Por su parte, la Sierra Patlachique tiene una menor altitud $(2700 \mathrm{msnm})$ y consiste de rocas volcánicas dacíticas, formadas durante el MiocenoPlioceno (Vázquez y Jaimes, 1989). La zona arqueológica de Teotihuacán se asienta sobre la planicie fluvial, constituida de materiales aluviales y laháricos (Tapia-Valera y LópezBlanco, 2002).

Los piedemontes de las sierras y el valle están disectados por barrancas profundas, modelados por cuatro ríos 

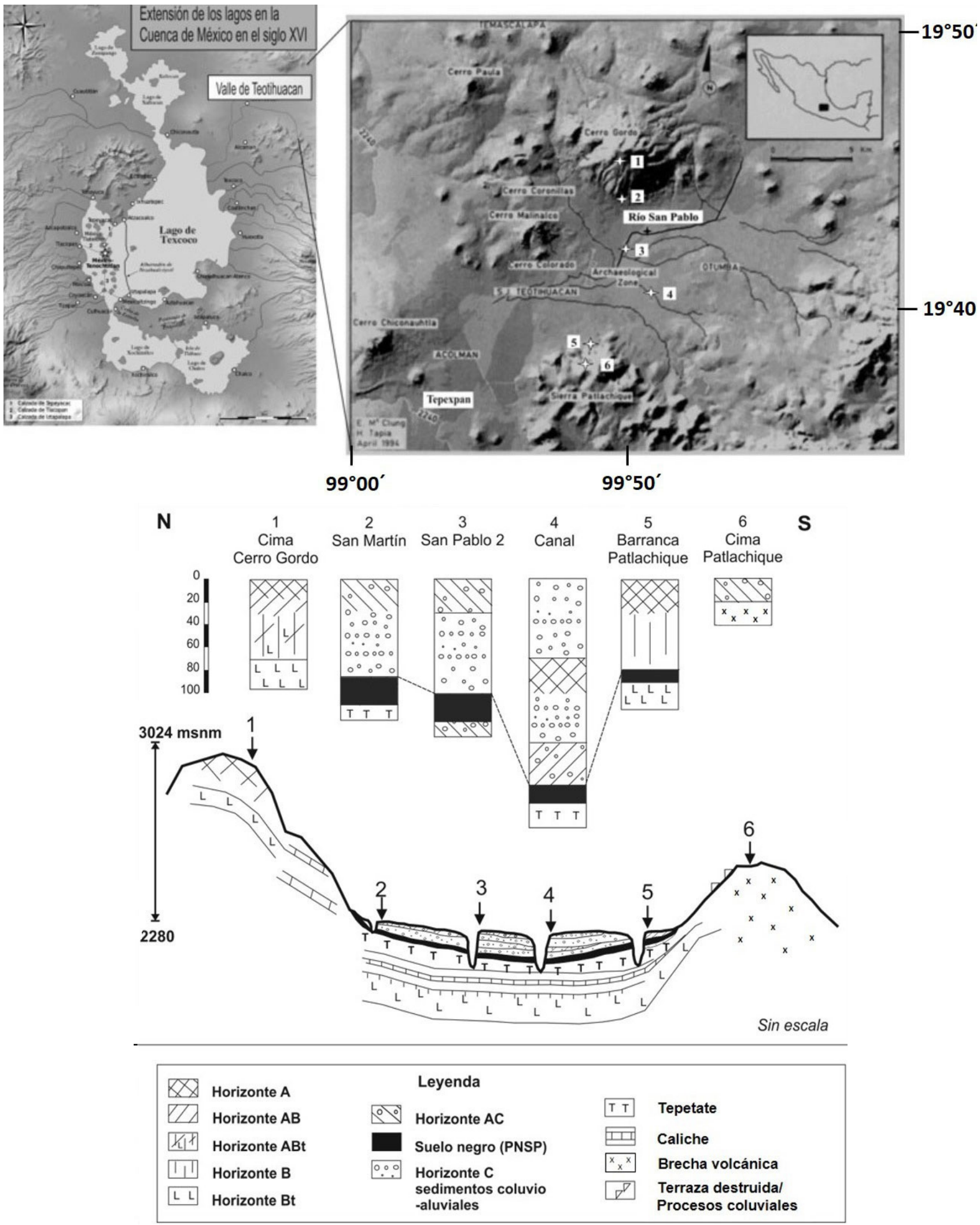

Figura 1. Localización del transecto N-S en el valle de Teotihuacán, mostrando los sitios de estudio. Esquema del perfil topográfico, en el que se presenta la morfología de los perfiles, así como la reconstrucción de los paleosuelos. 
principales: Los Estetes, El Muerto, El Soldado y Piedras Negras, los cuales se unen en la planicie para formar el río San Juan, el cual fue canalizado en la época prehispánica, para que cruzara la ciudad de Teotihuacán, a la altura de la Ciudadela (López-Austin, 1989).

La altitud del valle varía entre 2205 a 3050 m (Hernández, 2007). En consecuencia, se tienen variaciones climáticas que responden a los diferentes niveles altitudinales. En las zonas bajas (entre 2200 y $2800 \mathrm{msnm}$ ) se reporta un clima semiseco templado, con una precipitación que varía entre 500 - 600 mm (García, 1968, 1988). En las zonas altas (> $2800 \mathrm{msnm}$ ), se tiene un clima templado subhúmedo, con una precipitación que oscila entre 700 y $800 \mathrm{~mm}$. En general, el valle tiene una temperatura media anual que varía entre los 12 y $18^{\circ} \mathrm{C}$ (García, 1988).

Por su parte, también se registran cambios en la vegetación de acuerdo a la altitud (Rzedowski et al., 1964). En las partes bajas, hasta los $2750 \mathrm{msnm}$, domina el matorral xerófilo, como Opuntia streptacatha, Zaluzania augusta, Mimosa biuncifera y Yucca filifera. En las zonas más altas de las elevaciones que circundan al valle, particularmente en las laderas opuestas al valle, se encuentran bosques de encino (Rzedowski et al., 1964; Castilla y Tejero, 1983). También se localizan matorrales de encino, tanto en las laderas bajas como en las altas, así como cultivo de nopal (Opuntia), vegetación que atestigua la gran perturbación humana que ha existido en el lugar.

\section{Materiales y métodos}

\subsection{Los suelos del valle de Teotihuacán}

Las unidades de suelo que forman la cubierta edáfica moderna incluyen Phaeozems, Vertisoles, Cambisoles, Leptosoles y Fluvisoles. Estas unidades se distribuyen de la siguiente manera, de acuerdo con los estudios realizados por Gama-Castro et al. (2005) y Solleiro-Rebolledo et al. (2006):

Los Phaeozems son los suelos dominantes, ocupando el $46 \%$ del área, ubicándose en las laderas de los cerros y sierras, así como en los piedemontes. Los Vertisoles comprenden el $18 \%$ de la región. Se localizan en depresiones y en zonas de inclinación ligera ( $2 \%$ de pendiente). Los Cambisoles y Leptosoles ocupan, cada uno, el $13 \%$ de la zona y su distribución es heterogénea, en diferentes posiciones del relieve. Los Fluvisoles son los menos comunes $(10 \%)$ y se concentran en el fondo del valle, en las laderas del río San Juan.

\subsection{Paleosuelos del valle de Teotihuacán}

Durante los últimos años, se ha llevado a cabo un estudio intensivo de paleosuelos, sedimentos y arqueología en el valle de Teotihuacán (McClung de Tapia et al., 2003, 2005; Solleiro-Rebolledo et al., 2006, 2011; Sedov et al., 2010;
Sycheva et al., 2010; Sánchez et al., 2013; González et al., 2013). Durante esos estudios se han reconocido diversos paleosuelos que abarcan prácticamente los últimos 50000 años, cuyas características son:

- Paleosuelos arcillosos que forman una cubierta más o menos continua desde las posiciones elevadas, como el Cerro Gordo, los piedemontes de la Sierra Patlachique (Cabadas et al., 2005; SolleiroRebolledo et al., 2006) y el fondo del valle. Estos paleosuelos se formaron durante el Pleistoceno tardío y corresponden a Luvisoles crómicos en las partes altas de las sierras y Luvisoles estágnicos/ gléyicos en el fondo del valle. Esta diferencia, en las propiedades observadas, se debe a los procesos de saturación de agua en las posiciones más bajas, en contraste con el buen drenaje de los suelos en las sierras.

- Capas cementadas con carbonatos neoformados (horizontes petrocálcicos, denominados en este trabajo como caliches) incluidas en los paleosuelos arcillosos pleistocénicos (Solleiro-Rebolledo et al., 2011).

- Paleosuelos formados durante el Holoceno mediotardío, los cuales también son arcillosos, pero poseen horizontes orgánicos con propiedades vérticas. Estos paleosuelos también muestran una distribución continúa en diferentes posiciones, $y$ se encuentran relacionados con materiales arqueológicos de edad teotihuacana (Rivera-Uria et al., 2007; Solleiro-Rebolledo et al., 2011; Sánchez et al., 2013).

- Suelos y sedimentos formados en el último milenio.

\subsection{Trabajo de campo}

Para este trabajo, se llevó a cabo un transecto con dirección N-S, desde las posiciones elevadas del Cerro Gordo y la Sierra Patlachique (Figura 1), hasta las partes más bajas del valle. Se seleccionaron seis sitios a lo largo de la catena, ubicados preferentemente en cortes y barrancas que permitieran una mejor observación de la estratigrafía: (1) Cima Cerro Gordo; (2) San Martín; (3) San Pablo 2; (4) El Canal; (5) Barranca Patlachique; (6) Cima Patlachique.

En cada sitio, se describieron los perfiles, tomando en cuenta los criterios de la IUSS Working Group (2006) y se tomaron muestras inalteradas para realizar estudios micromorfológicos. Esta información sumada a la ya publicada, permitió reconstruir la distribución espacial y temporal de los suelos y paleosuelos.

\subsection{Micromorfología}

Para los estudios micromorfológicos, se seleccionaron muestras de algunos horizontes A que se supone coinciden con el Paleosuelo Negro San Pablo (PNSP), en diferentes posiciones geomorfológicas (San Martín y el Canal), para 
confirmar su correlación estratigráfica. Las muestras con estructura intacta se impregnaron con resina Crystal 40, se secaron, cortaron y pulieron en secciones delgadas, para su posterior estudio bajo un microscopio petrográfico Olympus.

\subsection{Cronología e isótopos estables de la secuencia}

Con el objetivo de afinar el esquema cronológicoestratigráfico, se usaron fechamientos por radiocarbono ya publicados en trabajos previos (Solleiro-Rebolledo et al., 2006, 2011; Sedov et al., 2010; Sánchez et al., 2013) así como nuevas edades obtenidas en carbonatos, en el LeibnizLaboratory for Radiometric Dating and Isotope Research, Kiel, Alemania (Tabla 1). La división cronológica de las unidades paleopedológicas se ha hecho, principalmente, con el apoyo de las fechas de radiocarbono de los carbonatos y costras de caliches, que cubren un lapso mayor a las determinadas por medio de otros materiales (humus de los horizontes orgánicos y extractos polínicos). En el mismo laboratorio, se obtuvo la composición de los isótopos estables de carbono $\left(\delta^{13} \mathrm{C}\right)$, usando el estándar VPB (Vienna Peedee Belemnite, \%o).

\section{Resultados}

\subsection{Morfología de los suelos y paleosuelos}

Los perfiles que se encuentran en el transecto N-S (Figura 1) muestran un conjunto de suelos superficiales y paleosuelos arcillosos, algunos de ellos con carbonatos secundarios, y sedimentos aluviales y coluviales. La descripción morfológica se presenta a continuación:

\subsubsection{Perfil 1. Cima Cerro Gordo}

Se encuentra en la cima del Cerro Gordo, junto a las torres de telecomunicaciones, a $3040 \mathrm{msnm}$. En este lugar aún se conserva el bosque de encino, aunque solo en parches. El micro-relieve es variado, cambiando de mesetas pequeñas de poca inclinación, a superficies con pendientes pronunciadas. El suelo estudiado presenta un perfil policíclico, constituido por horizontes $\mathrm{A}, \mathrm{AB}, \mathrm{ABt}$, $2 \mathrm{Bt}$, con un espesor de $80 \mathrm{~cm}$. Los horizontes que se presentan en los primeros $70 \mathrm{~cm}$ tienen una textura limo arcillosa, la cual contrasta con la aparición de un horizonte más compacto y más arcilloso (Figura 2).

\subsubsection{Perfil 2. San Martín}

Se encuentra en el piedemonte del Cerro Gordo, al oeste de una bajada de agua intermitente, proveniente del Cerro Gordo, que limita una serie de terrazas de cultivo, en descanso, a una altitud de $2950 \mathrm{msnm}$. En esta posición la pendiente es ligeramente convexa, con incisiones que producen barrancas profundas. El ancho y la profundidad de la barranca son de 20 y $1 \mathrm{~m}$, respectivamente. En el fondo de la barranca se encuentra un antiguo canal de riego. Domina la vegetación herbácea con abundancia de cactus, entre los que destaca Opuntia, sin embargo la cobertura no es completa, alcanzando 50 - 70 \%. En la superficie del terreno se observan abundantes fragmentos de rocas redondeadas y angulares, así como cerámica y herramientas de obsidiana.

El perfil estudiado es también policíclico, mostrando los siguientes horizontes Ap, AC, C, 2A, 2C. El suelo superficial (Ap, AC, C) muestra evidencias de cultivo, pero actualmente está mezclado con material coluvial cuyo depósito, es probable, se relacione con el manejo antrópico (por la reactivación de la terraza al dejarla abandonada). Su espesor es de $85 \mathrm{~cm}$ (Figura 3). Sobreyace a un paleosuelo con un horizonte $2 \mathrm{~A}$ de color muy oscuro, de $25 \mathrm{~cm}$ de espesor) que se ha relacionado con el Paleosuelo Negro San Pablo (PNSP) (Sánchez et al., 2013). El horizonte $2 \mathrm{C}$ muestra cierto endurecimiento, por lo que se le ha considerado como tepetate (material volcánico endurecido, pero no cementado).

\subsubsection{Perfil 3. San Pablo 2}

Este es un perfil ubicado en una posición cercana a la sección tipo (San Pablo), en donde se ha descrito con detalle el PNSP (Sánchez et al., 2013). Se encuentra en una barranca cortada por una corriente aluvial. La parte superior del perfil muestra un suelo con bajo desarrollo y mayor sedimentación, que está constituida únicamente por el horizonte $\mathrm{AC}(30 \mathrm{~cm})$, con pocos rasgos de pedogénesis (Figura 4). Por debajo se encuentra el horizonte 2C, un sedimento coluvio-aluvial grueso (70 cm de espesor), con intercalaciones de arena y grava. El paleosuelo negro (PNSP) se encuentra sepultado a $1 \mathrm{~m}$ de profundidad $\mathrm{y}$ solo se reconoce el horizonte $3 \mathrm{~A}$ que tiene un color oscuro (Figura 4) y que descansa directamente sobre un tepetate (horizonte 4C).

\subsubsection{Perfil 4. El canal}

Se localiza en la planicie aluvial del valle, junto a un canal de riego construido en la época prehispánica. Los primeros $70 \mathrm{~cm}$, desde la superficie, son ocupados por un sedimento mal clasificado, areno-limoso, y se le ha dividido en $\mathrm{C} 1$ y C2. C1 muestra un mayor grado de pedogénesis, que consiste, básicamente, en incorporación de materia orgánica y mayor agregación. En C2 destaca la presencia de laminaciones. Dos paleosuelos subyacen a este sedimento, constituidos por los horizontes $2 \mathrm{~A}, 2 \mathrm{C}, 3 \mathrm{AE}, 3 \mathrm{ABtss}$ y $4 \mathrm{C}$ (Figura 5). El primer paleosuelo tiene $60 \mathrm{~cm}$ de espesor $\mathrm{y}$ su desarrollo es débil. El segundo paleosuelo (3A, 3ABtss) muestra mayor grado de desarrollo y se correlaciona con el PNSP. En la base del perfil se presenta un tepetate (horizonte 4C).

\subsubsection{Perfil 5. Barranca Patlachique}

Se localiza sobre la parte media de la Sierra Patlachique, justo a la orilla de una corriente de agua meandriforme, ubicándose en la parte erosiva del meandro, que forma una 
Tabla 1. Edades de radiocarbono y valores de isótopos estables de carbono obtenidas en carbonatos y materia orgánica de los paleosuelos del valle de Teotihuacán.

\begin{tabular}{|c|c|c|c|c|c|c|c|c|}
\hline $\begin{array}{c}\text { Código de } \\
\text { Laboratorio }\end{array}$ & Localidad & Horizonte & $\begin{array}{l}\text { Prof.* } \\
\text { cm }\end{array}$ & Material & $\begin{array}{c}\text { Edad } \\
\text { Años AP }\end{array}$ & $\begin{array}{c}\text { Edad calibrada } \\
\text { Años AP }\end{array}$ & $\begin{array}{c}\delta^{13} \mathrm{C} \\
\%\end{array}$ & Ref. \\
\hline Beta 219962 & $\begin{array}{c}\text { San Pablo } \\
19^{\circ} 42^{\prime} 33.17^{\prime \prime} \mathrm{N} \\
98^{\circ} 49^{\prime} 19.02^{\prime \prime} \mathrm{W}\end{array}$ & $3 \mathrm{~A}$ & 90 & $\begin{array}{l}\text { Materia orgánica } \\
\text { quemada }\end{array}$ & $490 \pm 40$ & $529 \pm 15$ & -22 & 1 \\
\hline Beta 219963 & $\begin{array}{c}\text { San Pablo } \\
19^{\circ} 42^{\prime} 33.17^{\prime \prime} \mathrm{N} \\
98^{\circ} 49^{\prime} 19.02^{\prime \prime} \mathrm{W}\end{array}$ & $4 \mathrm{~A}$ & 125 & $\begin{array}{l}\text { Materia orgánica } \\
\text { quemada }\end{array}$ & $620 \pm 40$ & $608 \pm 39$ & -22 & 2 \\
\hline Beta 210760 & $\begin{array}{c}\text { San Pablo } \\
19^{\circ} 42^{\prime} 30.9^{\prime \prime} \mathrm{N} \\
98^{\circ} 49^{\prime} 15.2^{\prime \prime} \mathrm{W}\end{array}$ & $4 \mathrm{~A}$ & 155 & Materia orgánica & $2140 \pm 60$ & $2162 \pm 110$ & -16.7 & 1 \\
\hline KIA 42668 & $\begin{array}{l}\text { Cerro Gordo } \\
19^{\circ} 44^{\prime} 35^{\prime \prime} \mathrm{N} \\
98^{\circ} 48^{\prime} 35^{\prime \prime} \mathrm{W}\end{array}$ & Ck1 & 50 & $\begin{array}{c}\text { Carbonatos sobre } \\
\text { roca }\end{array}$ & $2475 \pm 30$ & $2580 \pm 95$ & -5.52 & 3 \\
\hline Beta 261617 & $\begin{array}{c}\text { San Pablo } \\
19^{\circ} 42^{\prime} 33.17^{\prime \prime N} \\
98^{\circ} 49^{\prime} 19.02^{\prime \prime} \mathrm{W}\end{array}$ & 5 ABtss & 205 & Materia orgánica & $2980 \pm 40$ & $3166 \pm 69$ & -16.7 & 2 \\
\hline KIA 42675 & $\begin{array}{c}\text { Maseca } \\
19^{\circ} 39^{\prime} 35^{\prime \prime} \mathrm{N} \\
98^{\circ} 49^{\prime} 16.1^{\prime \prime} \mathrm{W}\end{array}$ & $\mathrm{Bk}$ & 40 & $\begin{array}{l}\text { Carbonatos } \\
\text { diseminados }\end{array}$ & $3655 \pm 30$ & $3996 \pm 62$ & -2.83 & 3 \\
\hline Beta 307550 & $\begin{array}{c}\text { La Ventilla } \\
19^{\circ} 40^{\prime} 51.1^{\prime \prime} \mathrm{N} \\
98^{\circ} 51^{\prime} 20.3^{\prime \prime} \mathrm{W}\end{array}$ & Btkb & 180 & $\begin{array}{l}\text { Carbonatos } \\
\text { diseminados }\end{array}$ & $5080 \pm 30$ & $5831 \pm 57$ & -6.7 & 4 \\
\hline GIN 12352 & $\begin{array}{l}\text { Cerro Gordo } \\
19^{\circ} 44^{\prime} 55^{\prime \prime} \mathrm{N} \\
98^{\circ} 49^{\prime} 24^{\prime \prime} \mathrm{W}\end{array}$ & $2 \mathrm{~A}$ & 71 & Materia orgánica & $13340 \pm 300$ & $16215 \pm 541$ & -20.9 & 2 \\
\hline Beta 73337 & $\begin{array}{l}\text { Cerro Gordo } \\
19^{\circ} 44^{\prime} 55^{\prime \prime} \mathrm{N} \\
98^{\circ} 49^{\prime} 24^{\prime \prime} \mathrm{W}\end{array}$ & $2 \mathrm{Bt}$ & 97 & Materia orgánica & $18740 \pm 150$ & $22422 \pm 65$ & & 5 \\
\hline KIA 42677 & $\begin{array}{c}\text { Maseca } \\
19^{\circ} 39^{\prime} 35^{\prime \prime} \mathrm{N} \\
98^{\circ} 49^{\prime} 16.1^{\prime \prime} \mathrm{W}\end{array}$ & 2Btg & 120 & $\begin{array}{c}\text { Fractura rellena de } \\
\text { carbonatos }\end{array}$ & $18730 \pm 130$ & $22410 \pm 353$ & $-4,28$ & 3 \\
\hline Beta 293587 & $\begin{array}{c}\text { San Pablo } \\
19^{\circ} 42^{\prime} 30.9^{\prime \prime} \mathrm{N} \\
98^{\circ} 49^{\prime} 15.2^{\prime \prime} \mathrm{W}\end{array}$ & $5 \mathrm{Ck}$ & 60 & Caliche & $20990 \pm 100$ & $25132 \pm 373$ & -6.7 & 4 \\
\hline Beta 73338 & $\begin{array}{l}\text { Cerro Gordo } \\
19^{\circ} 44^{\prime} 55^{\prime \prime} \mathrm{N} \\
98^{\circ} 49^{\prime} 24^{\prime \prime} \mathrm{W}\end{array}$ & $3 \mathrm{Bt}$ & 110 & Materia orgánica & $22670 \pm 290$ & $27326 \pm 76$ & & 5 \\
\hline Beta 210760 & $\begin{array}{c}\text { San Pablo } \\
19^{\circ} 42^{\prime} 30.9^{\prime \prime} \mathrm{N} \\
98^{\circ} 49^{\prime} 15.2^{\prime \prime} \mathrm{W}\end{array}$ & $6^{\prime} \mathrm{Bk}$ & 240 & Caliche & $24070 \pm 350$ & $28914 \pm 480$ & -5.3 & 2 \\
\hline KIA 42674 & $\begin{array}{c}\text { Maseca } \\
19^{\circ} 39^{\prime} 35^{\prime \prime} \mathrm{N} \\
98^{\circ} 49^{\prime} 16.1^{\prime \prime} \mathrm{W}\end{array}$ & 3Btg & 150 & $\begin{array}{c}\text { Fractura rellena de } \\
\text { carbonatos }\end{array}$ & $26520+250 /-240$ & $31261 \pm 381$ & -4.03 & 3 \\
\hline KIA 40702 & $\begin{array}{l}\text { Cerro Gordo } \\
19^{\circ} 44^{\prime} 35^{\prime \prime} \mathrm{N} \\
98^{\circ} 48^{\prime} 35^{\prime \prime} \mathrm{W}\end{array}$ & $3 \mathrm{Ck}$ & 240 & Caliche & $26210+230 /-220$ & $31081 \pm 367$ & -5.46 & 3 \\
\hline KIA 40701 & $\begin{array}{l}\text { Cerro Gordo } \\
19^{\circ} 44^{\prime} 35^{\prime \prime} \mathrm{N} \\
98^{\circ} 48^{\prime} 35^{\prime \prime} \mathrm{W}\end{array}$ & $2 \mathrm{Ck}$ & 80 & Caliche & $31250+390 /-370$ & $35267 \pm 463$ & -5.35 & 3 \\
\hline KIA 42661(1) & $\begin{array}{c}\text { San Pablo } \\
19^{\circ} 42^{\prime} 30.9^{\prime \prime} \mathrm{N} \\
98^{\circ} 49^{\prime} 15.2^{\prime \prime} \mathrm{W}\end{array}$ & $6 \mathrm{Ck}$ & 280 & $\begin{array}{c}\text { Fracturas rellenas } \\
\text { con carbonatos }\end{array}$ & $34430+720 /-660$ & $39612 \pm 1013$ & -4.07 & 3 \\
\hline KIA 42661(2) & $\begin{array}{c}\text { San Pablo } \\
19^{\circ} 42^{\prime} 30.9^{\prime \prime} \mathrm{N} \\
98^{\circ} 49^{\prime} 15.2^{\prime \prime} \mathrm{W}\end{array}$ & $6 \mathrm{Ck}$ & 280 & $\begin{array}{c}\text { Fracturas rellenas } \\
\text { con carbonatos }\end{array}$ & $36780+940 /-840$ & $41663 \pm 640$ & -7.68 & 3 \\
\hline
\end{tabular}

* Profundidad desde la superficie.

${ }^{1}$ Rivera-Uria et al. (2007); ${ }^{2}$ Solleiro-Rebolledo et al. (2011); ${ }^{3}$ Este trabajo; ${ }^{4}$ Sánchez et al. (2013); ${ }^{5}$ McClung et al. (2003). 
Perfil 1. Cima Cerro Gordo. 19०45'16.3" N; 9849'31.97"W; 3040 msnm

\begin{tabular}{|c|c|c|c|}
\hline 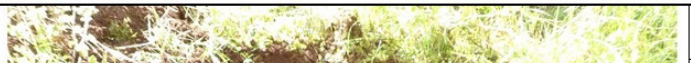 & Horizonte & $\begin{array}{c}\text { Prof. } \\
\text { cm }\end{array}$ & Descripción \\
\hline . & $\mathrm{Ah}$ & $0-15$ & $\begin{array}{l}\text { Color pardo oscuro; textura limo- arcillosa; friable; estructura } \\
\text { granular bien desarrollada; abundantes coprolitos, raíces y poros } \\
\text { biogénicos; se presentan minerales volcánicos frescos. }\end{array}$ \\
\hline & $\mathrm{AB}$ & $15-30$ & $\begin{array}{l}\text { Color pardo oscuro; textura arcillo- limosa; estructura en } \\
\text { bloques subangulares medianos ( } 3-5 \mathrm{~cm}) \text {, que rompen a } \\
\text { granular; porosidad de tipo biogénica con abundantes coprolitos, } \\
\text { aunque aquí la densidad de raíces es menor. }\end{array}$ \\
\hline & $\mathrm{ABt}$ & $30-70$ & $\begin{array}{l}\text { Color pardo rojizo-grisáceo; textura arcillo-limosa; estructura } \\
\text { en bloques medianos; sub angulares; bien desarrollada; se } \\
\text { observan cutanes de arcilla de color oscuro (con humus); } \\
\text { bioturbación con restos coprogénicos y raíces; el límite con el } \\
\text { horizonte subyacente es abrupto. }\end{array}$ \\
\hline 60 & $2 \mathrm{Bt}$ & $70-80$ & $\begin{array}{l}\text { Color rojo parduzco; textura arcillosa; estructura en bloques } \\
\text { angulares bien desarrollados; con discontinuos y zonales cutanes } \\
\text { de arcilla y manganeso; de color negro brillante; no se observan } \\
\text { raíces. }\end{array}$ \\
\hline
\end{tabular}

Figura 2. Fotografía y descripción morfológica del perfil 1 Cerro Gordo

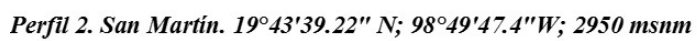

\begin{tabular}{|c|c|c|c|}
\hline 5 & Horizonte & $\begin{array}{l}\text { Prof } \\
(\mathrm{cm})\end{array}$ & Descripción \\
\hline 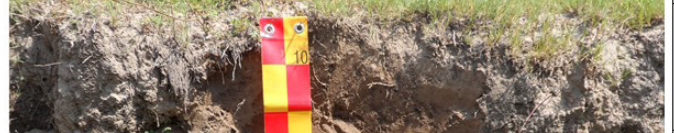 & Ap-AC & $0-30$ & $\begin{array}{l}\text { Color pardo claro; textura limo-arenosa; estructura granular } \\
\text { poco desarrollada; friable; con presencia de raíces; } \\
\text { abundante cerámica y fragmentos de roca, algunos de los } \\
\text { cuales efervescen con } \mathrm{HCl} \text {, en su superficie. }\end{array}$ \\
\hline$\frac{x^{2}+2 x}{4 x^{2}}$ & $\mathrm{C}$ & $30-85$ & $\begin{array}{l}\text { Color pardo claro, más claro que el anterior; textura areno- } \\
\text { limosa; sin estructura; es un sedimento de tipo coluvial con } \\
\text { intercalaciones de arena de color ocre, laminadas; escasas } \\
\text { raíces; la superficie de las rocas muestran una ligera } \\
\text { efervescencia. El límite con el horizonte subyacente es } \\
\text { abrupto y plano (erosional). }\end{array}$ \\
\hline se. & $2 \mathrm{~A}$ & $85-110$ & $\begin{array}{l}\text { Color pardo muy oscuro; textura limo - arcillosa; } \\
\text { estructura en bloques sub angulares; de medianos a } \\
\text { pequeños (menores de } 3 \mathrm{~cm}) \text {; consistencia friable no se } \\
\text { observan raíces. Hay algunas gravas pequeñas }(0.5 \mathrm{~cm}) \text { que } \\
\text { no efervescen. }\end{array}$ \\
\hline & $2 \mathrm{C}$ & $>110$ & Color pardo; textura limosa; sin estructura. Tepetate. \\
\hline
\end{tabular}

Figura 3. Fotografía y descripción morfológica del perfil 2 San Martín.

Perfil 3. San Pablo 2. 1942'31.7”N 98०49'00" $2308 \mathrm{msnm}$

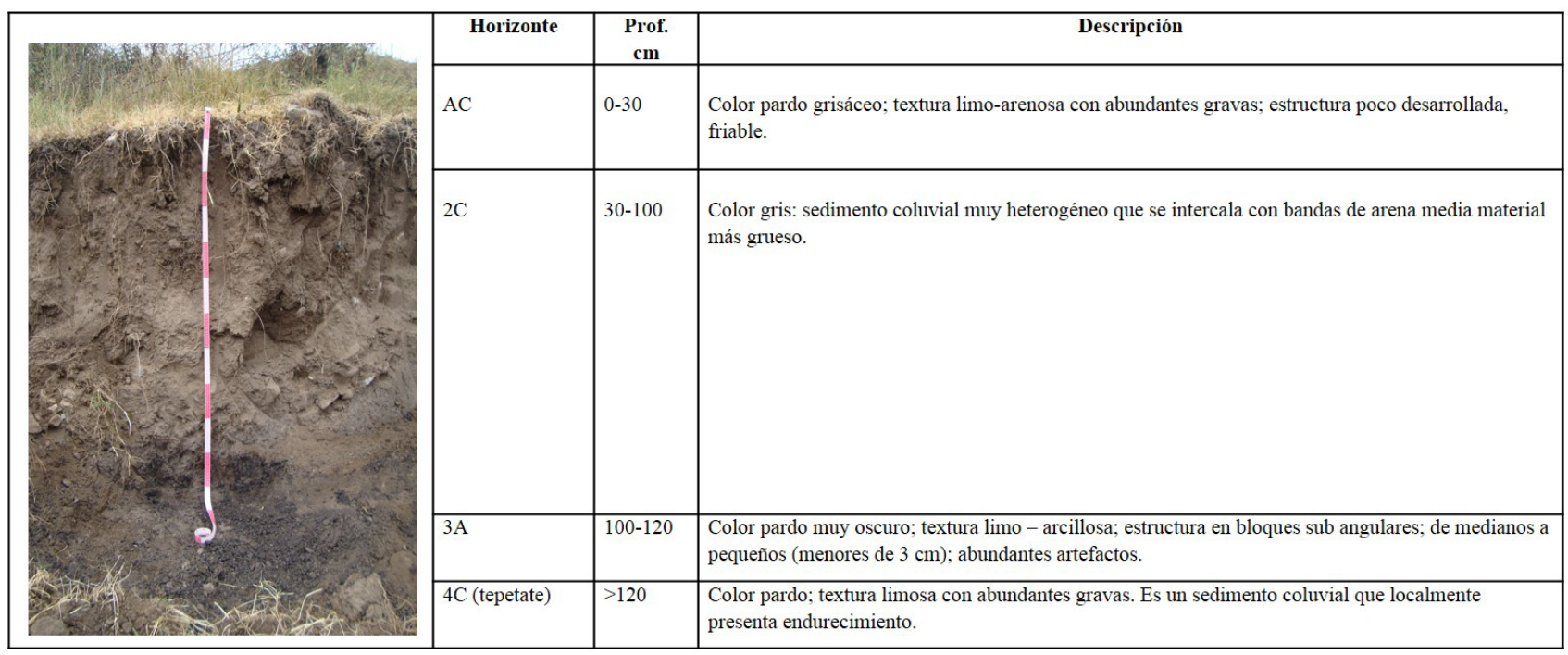

Figura 4. Fotografía y descripción morfológica del perfil 3, San Pablo 2. 
barranca. Esta barranca inicia en las laderas de la sierra y termina en el valle. Tiene forma de U, con $40-50 \mathrm{~m}$ de ancho, entre ladera y ladera, y $8-10 \mathrm{~m}$ de profundidad. En el fondo de la barranca se encuentra un tepetate formado por sedimentos coluviales.

$\mathrm{Al}$ igual que en los casos anteriores, se trata de un suelo policíclico (Figura 6), con tres ciclos de formación de suelo: el suelo moderno de $80 \mathrm{~cm}$ de espesor con horizontes $\mathrm{Ah}$, $\mathrm{B} 1$, B2, es de textura limosa; el primer paleosuelo muestra un solo horizonte $2 \mathrm{AB}$, de $10 \mathrm{~cm}$ de espesor, y textura más fina, que se correlaciona con el PNSP. En el tercer paleosuelo se observa solo el horizonte $3 \mathrm{Bt}$ de $20 \mathrm{~cm}$ de espesor.

Perfil 4. El Canal 19 40'12.5"N; 98 50'40.0"W; $2280 \mathrm{msnm}$

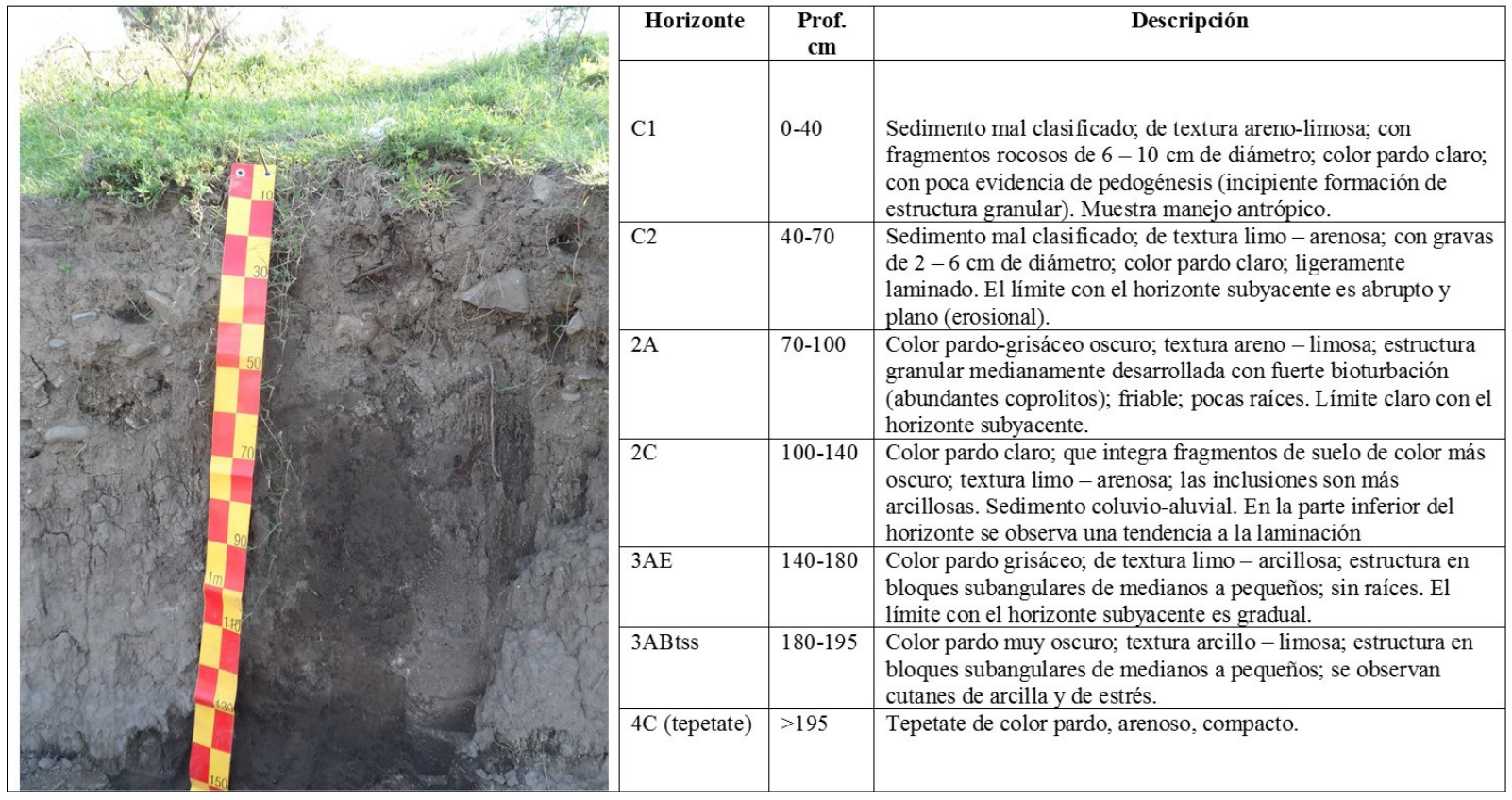

Figura 5. Fotografía y descripción morfológica del perfil 4, Canal.

\section{Perfil 5. Barranca Patlachique 19³8'13.1"N; 9848'40.2"W}

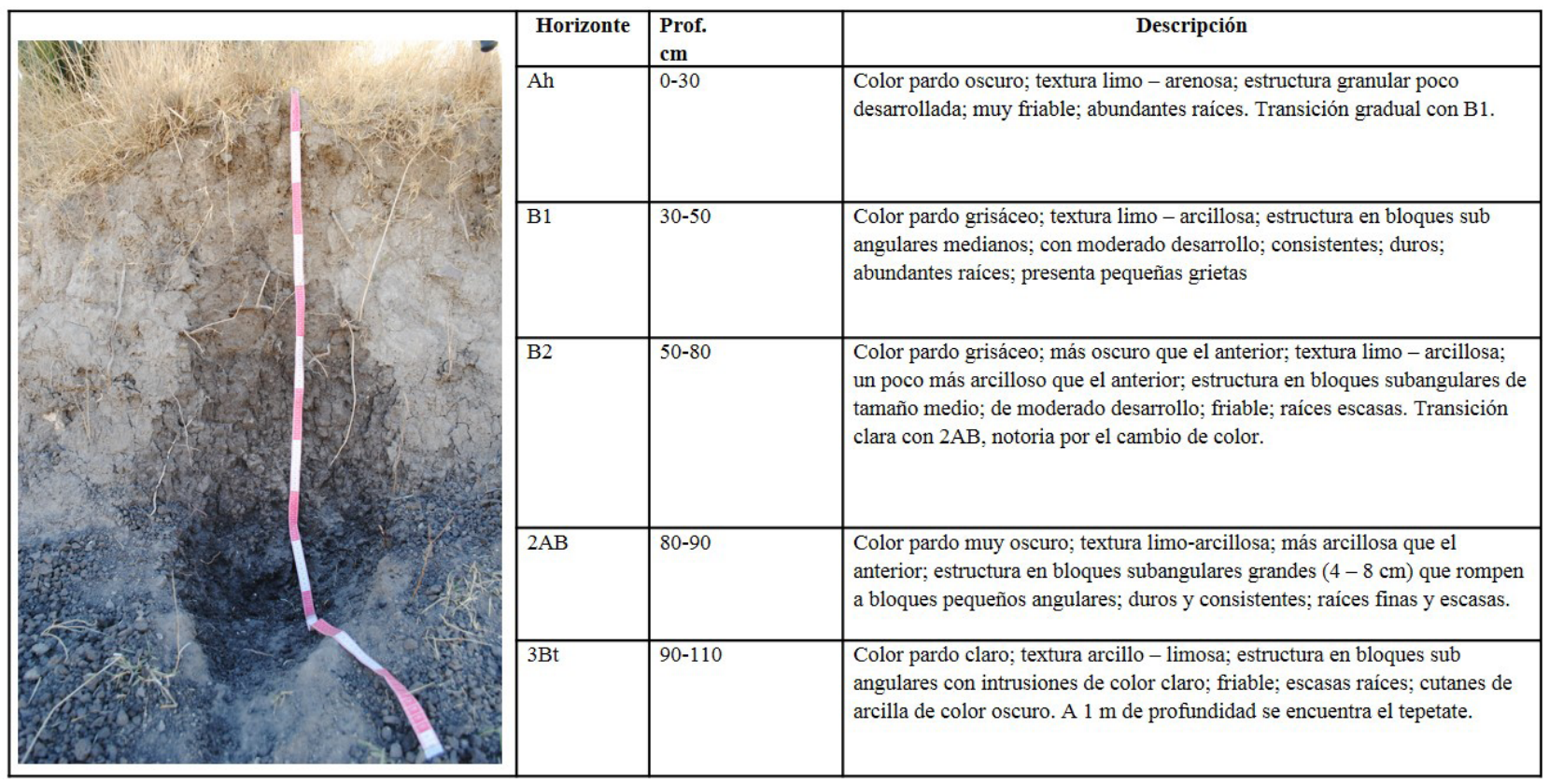

Figura 6. Fotografía y descripción morfológica del perfil 5, Sierra Patlachique. 


\subsubsection{Perfil 6. Cima Patlachique}

Este perfil de escaso desarrollo, se encuentra en la cima de una de las elevaciones que conforman a la Sierra Patlachique. El talud de la sierra está formado por una serie de terrazas antiguas, de época teotihuacana, de 5- $7 \mathrm{~m}$ de ancho (aunque las terrazas más bajas tienen hasta $30 \mathrm{~m}$ de anchura). Todavía se conservan restos de los muros de piedra que limitan las terrazas, aunque ya están muy destruidas por procesos de sedimentación y erosión (Figura 1). El suelo que se encuentra en esta posición geomorfológica es de escaso desarrollo y consiste únicamente de un horizonte $\mathrm{A}$, de 20 $\mathrm{cm}$ de espesor, que descansa directamente sobre una brecha volcánica (Figura 7).

\subsection{Micromorfología de los horizontes A de color oscuro}

A nivel microscópico, los horizontes A analizados, del PNSP, tienen un conjunto de características, similares a las establecidas anteriormente en el perfil San Pablo (Rivera-Uria et al., 2007; Sedov et al., 2009; Sánchez et al., 2013). Particularmente destaca la pigmentación obscura de la matriz, la presencia de componentes finos y una microestructura en forma de bloques angulares muy compactos, formados por fracturas de configuración vértica. Además de estos rasgos, llama la atención, la abundancia de cutanes arcillosos de iluviación, que se desarrollan en fracturas y en canales. A pesar de las similitudes, estos horizontes desarrollados en diferentes posiciones geomorfológicas muestran ciertos rasgos específicos que se desvían de los observados en el perfil San Pablo (RiveraUria et al., 2007; Sánchez et al., 2013). En el perfil 2 San Martin, en donde el horizonte $2 \mathrm{~A}$ del paleosuelo se encuentra sepultado a $80 \mathrm{~cm}$ de la superficie, se encontró una mayor cantidad de componentes gruesos (partículas de arena y hasta grava) representados por minerales volcánicos como plagioclasas, piroxenos, fragmentos de roca andesítica y vidrio volcánico poroso (pómez). Las partículas de pómez muestran rasgos de intemperismo moderado, ya que la parte periférica de las partículas está sustituida por arcilla. Estos rasgos se identifican claramente en las láminas delgadas dados los colores de interferencia, observados con nícoles cruzados (Figuras 8a y 8b). La microestructura de este horizonte no es uniforme: además de los bloques compactos, hay áreas con alta porosidad y agregados formando gránulos pequeños de origen coprogénico. Los rellenos de estos gránulos frecuentemente se asocian con poros $\mathrm{y} / \mathrm{o}$ canales biogénicos (Figura 8c). También en algunos poros identificamos recubrimientos de carbonatos neoformados, en forma de micrita (calcita microcristalina) (Figura 8d). Asimismo, se presentan cutanes de arcilla, los cuales se encuentran en áreas claramente diferentes de aquéllas en las que se tiene la calcita neoformada.

En el perfil 4, el Canal, en el horizonte 2A sepultado se observaron microlaminaciones de origen sedimentario. En la matriz domina el material fino, pero por ella atraviesan capas microscópicas de arena fina (Figura 8e). Además, en los agregados granulares, las capas microscópicas están parcialmente soldadas y forman áreas más porosas dentro de la matriz compacta (Figura 8f). Otra característica específica observada en este horizonte es el mejor desarrollo de los edaforrasgos relacionados con la illuviación de arcilla. Los gruesos rellenos de arcilla ocupan fracturas y poros. Bajo nícoles cruzados, estos rellenos tienen alta birrefrigencia, lo que indica que las partículas arcillosas están orientadas. Sin embargo, la distribución de los colores de interferencia no es uniforme, de manera que el relleno ha sufrido deformación y desorientación, posiblemente por el desarrollo de los rasgos vérticos (Figuras $8 \mathrm{~g}$ y $8 \mathrm{~h}$ ).

\subsection{Cronología de los paleosuelos}

De acuerdo con el conjunto de edades obtenidas para los carbonatos de la región de Teotihuacán (Tabla 1) así como a la información generada en trabajos previos (Solleiro-Rebolledo et al., 2011; Sánchez et al., 2013), es posible agrupar a los paleosuelos en los siguientes periodos cronológicos:

\subsubsection{Paleosuelos del Pleistoceno, fase I}

Estos palesuelos abarcan un periodo entre $16000 \mathrm{y}$ 50000 años AP. En este periodo se tiene un conjunto de edades de radiocarbono $\left({ }^{14} \mathrm{C}\right)$, que varían de 41000 a 16000

Perfil 6. Cima Patlachique. $19^{\circ} 37^{\prime} 55.2 " N$; 98 $48^{\prime} 31.18^{\prime \prime} \mathrm{W} ; 2700 \mathrm{msnm}$

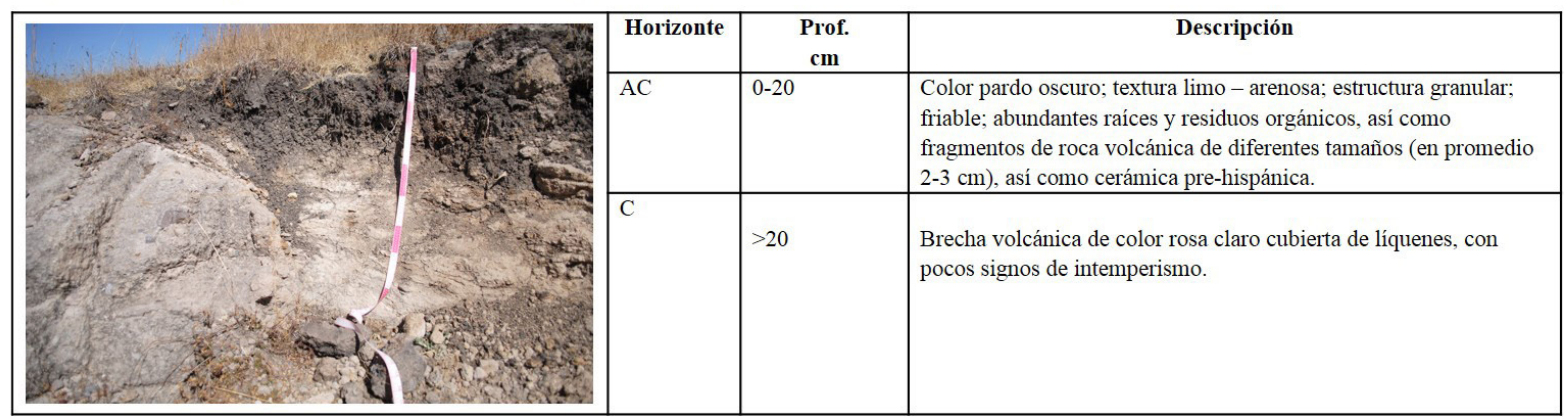

Figura 7. Fotografía y descripción morfológica del perfil 6, Cima de la Sierra Patlachique. 
a
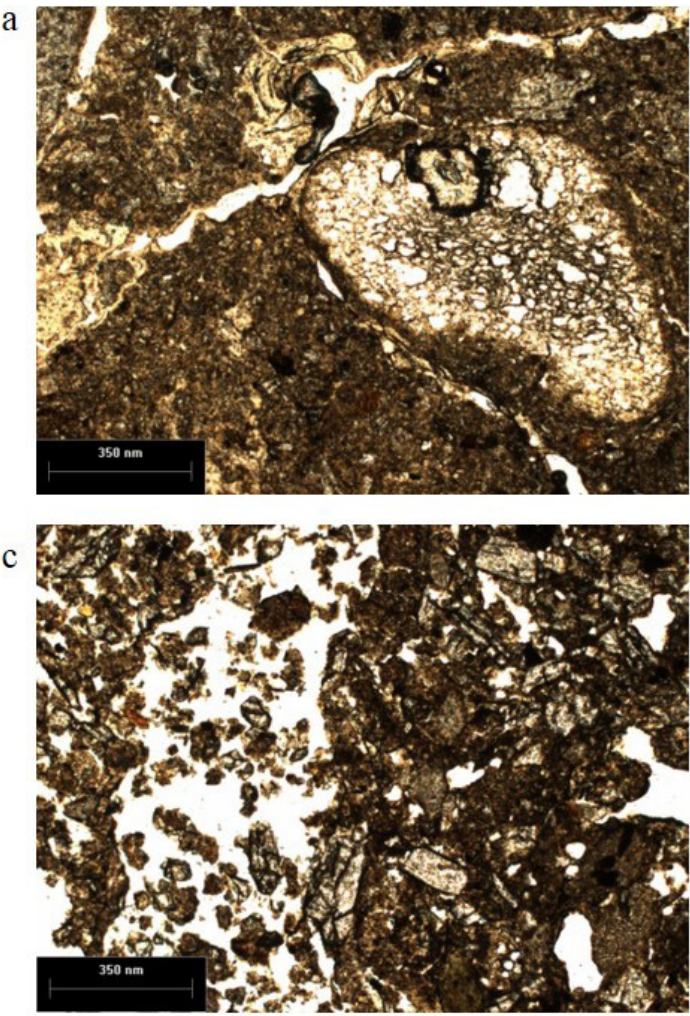

e

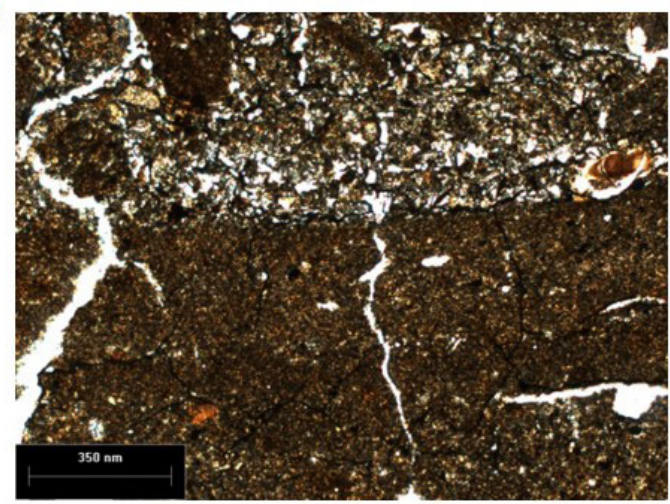

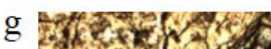

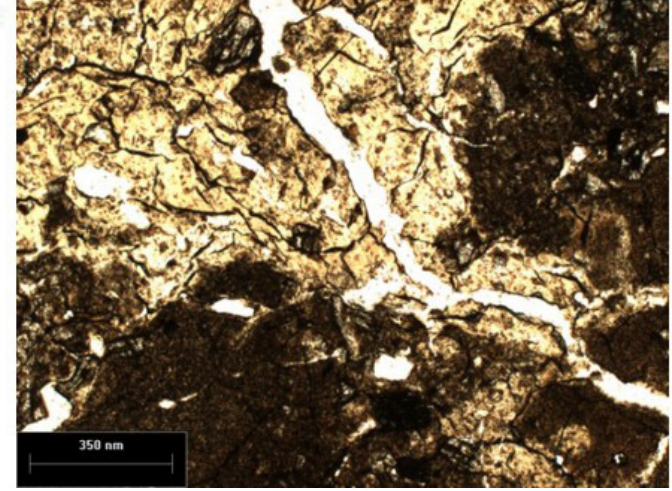

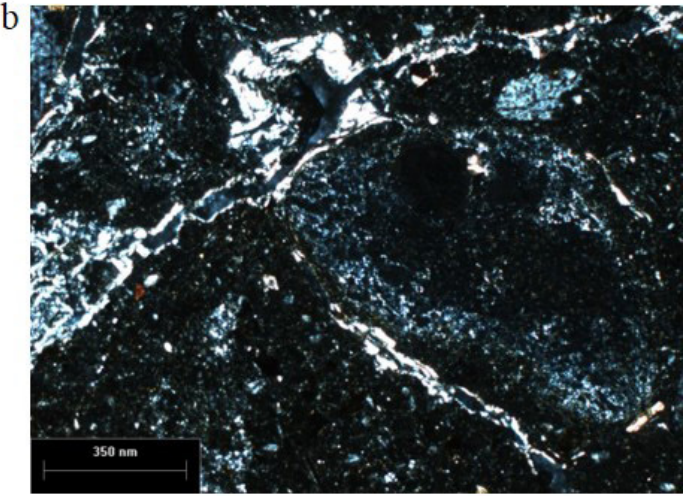

d

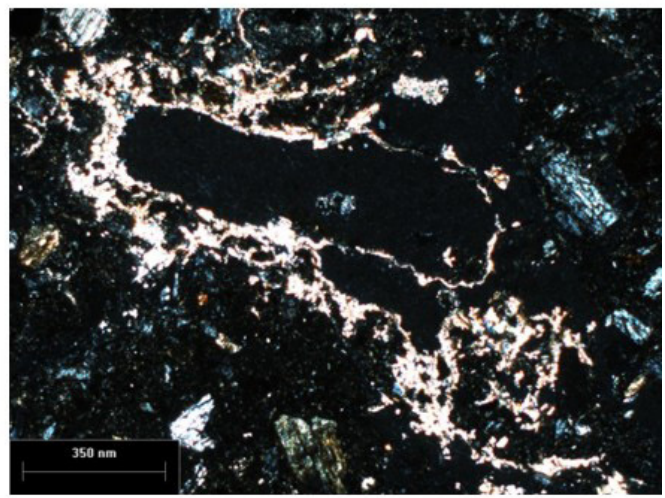

f
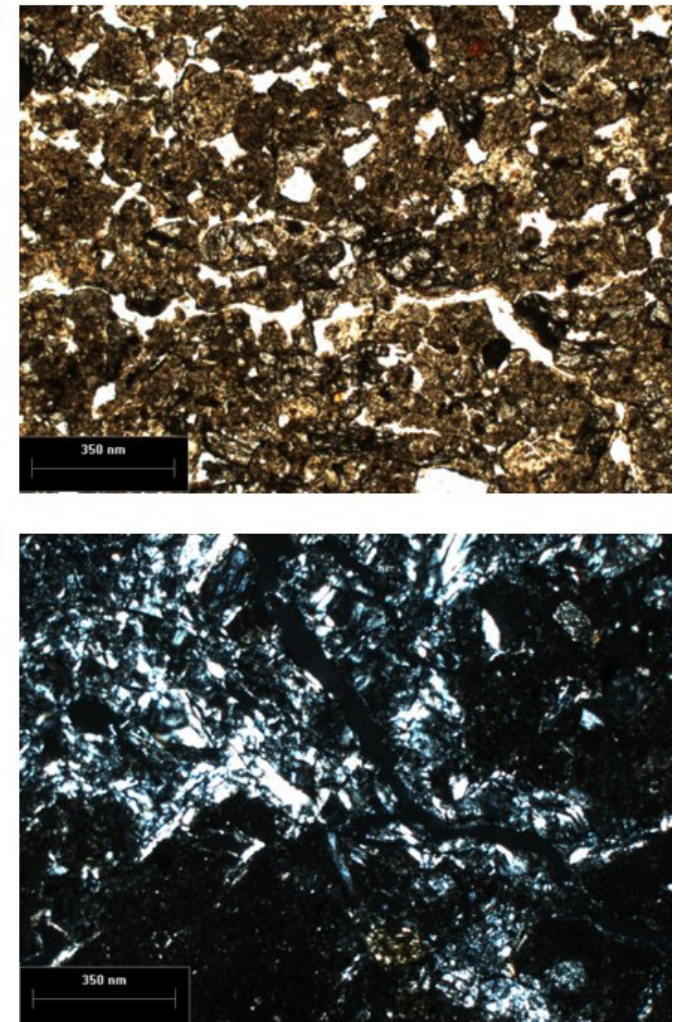

Figura 8. Micromorfología de los horizontes húmicos del Paleosuelo Negro San Pablo en perfiles selectos. a. Grano de pómez intemperizado, perfil 2 San Martín, luz normal polarizada. b. Mismo que a) pero con nícoles X. Nótese que la parte periférica del grano de la pómez tiene colores de interferencia por sustitución con arcilla; también es notable la presencia de colores de interferencia del cután de arcilla en el poro ubicado en la parte superior. c. Porocanal biogénico con un relleno poroso de microagregados granulares de origen coprogénico, perfil 2 San Martín, luz normal polarizada. d. Concentración de micrita, perfil 2 San Martín, nícoles X. e. Microlaminación, perfil 4, El Canal, luz normal polarizada. f. Capa constituida por agregados granulares, parcialmente soldados (la mitad superior de la imagen), perfil 4. g. Relleno compacto de arcilla iluviada en forma de abanico, perfil 4, luz normal polarizada. h. Mismo que h), nícoles X; nótese el patrón de mosaico de los colores de interferencia en el relleno de arcilla. 
años, obtenidas tanto en los carbonatos secundarios, como en materia orgánica (Tabla 1). Estos materiales carbonatados se localizan en Cerro Gordo, Maseca y San Pablo.

Los paleosuelos se caracterizan por presentar perfiles tipo $\mathrm{E} / \mathrm{EB} / \mathrm{Bt} / \mathrm{Btg} / \mathrm{Bgk} / \mathrm{C}$, aunque con frecuencia aparecen truncados por la erosión, de manera que solo se observan horizontes Bg y C. Únicamente en el Cerro Gordo, se ha reportado un perfil completo de un Luvisol crómico sepultado, con horizontes $2 \mathrm{~A}, 2 \mathrm{Bt}, 3 \mathrm{Bt}$, cuya edad oscila entre 16000 y 27000 años (Solleiro-Rebolledo et al., 2006), de acuerdo a los fechamientos de la materia orgánica en cada horizonte (Tabla 1). En general, la textura de estos paleosuelos es limo-arcillosa, con contenidos de limo que varían de 60 a $80 \%$. La fracción arcilla alcanza hasta $20 \%$ en los horizontes $\mathrm{Bt}$, los cuales poseen gruesos cutanes de arcilla y rasgos reductomórficos, caracterizados por mostrar colores gris parduzco, con abundantes moteados (gris acero y negro) y abundantes cutanes de Mn dendrítico.

Los paleosuelos ubicados en las partes bajas y en los piedemontes (San Pablo y Maseca) están cortados o intercalados por carbonatos cuyas edades van de 39000 a 41000 años A.P., y de 22000 a 29000 años A.P. (Tabla 1). En este sentido, si los carbonatos cortan a los paleosuelos arcillosos, éstos se formaron primero y representan las fases iniciales de pedogénesis. En las posiciones elevadas, como en el Cerro Gordo, la matriz de los paleosuelos está libre de carbonatos, pero se observan caliches intercalados en los sedimentos o sobre las rocas (Figura 9). Las edades de estos materiales son 35267 y 31082 años A.P. (Tabla 1).

Los valores de isótopos estables de carbono de los carbonatos pedogenéticos varían entre - 4.03 y - $7.68 \%$, este último valor corresponde con el caliche más antiguo (Tabla 1). La materia orgánica del horizonte $2 \mathrm{~A}$ sepultado tiene un valor de - $20.9 \%$ (Lounejeva et al., 2006).

\subsubsection{Paleosuelos y sedimentos del Pleistoceno, fase II}

Estos materiales se desarrollan entre 14000 y 11000 años. Prácticamente no se tiene registro de paleosuelos que comprendan estas edades en el fondo del Valle de Teotihuacán, o por lo menos los fechamientos obtenidos no comprenden este periodo (McClung de Tapia et al., 2005). Sin embargo, en San Pablo, se presentan Gleysoles cortados por canales aluviales (Solleiro-Rebolledo et al., 2011). Por lo tanto, se considera que en esta fase hay una mayor sedimentación que pedogénesis. Es por ello que se ha separado en un intervalo cronológico diferente, ya que cambian las condiciones de formación de suelos.

\subsubsection{Paleosuelos del Holoceno, fase I}

En estos paleosuelos se incluye a aquellos formados en el Holoceno temprano al tardío (aproximadamente entre 11000 y 1000 años A.P.). En este caso se integran paleosuelos arcillosos, poligenéticos que cubren un lapso considerable de pedogénesis, pero que han sido afectados por procesos diversos, naturales y antrópicos. Las edades que se tienen para establecer el marco cronológico de estos paleosuelos se concentran en el Holoceno medio (5831 - 3999 años), obtenidas en carbonatos, y de 3166 a 2162 años (Tabla 1), a partir de materiales orgánicos (Rivera-Uria et al., 2007; Solleiro-Rebolledo et al., 2011; Sánchez et al., 2013). A

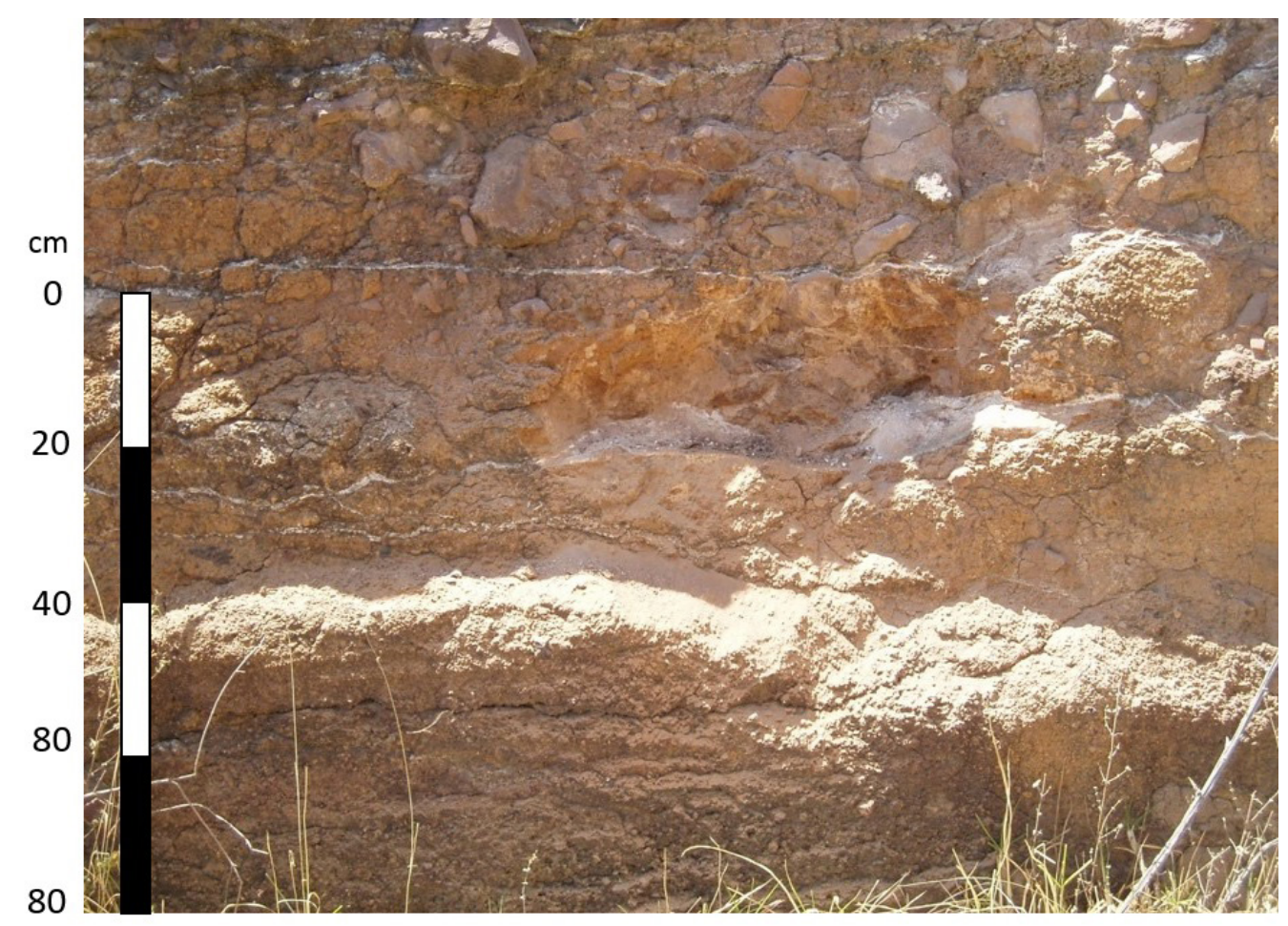

Figura 9. Caliches con edad por radiocarbono de 35,267 \pm 463 años AP sobre rocas en el Cerro Gordo. 
pesar de no tener edades relacionadas al Holoceno temprano, los carbonatos fechados (Tabla 1) se encuentran en el horizonte 5ABtss, sobre cutanes de arcilla, lo que demuestra que la iluviación es un proceso previo y cubre un intervalo de tiempo mayor.

Los valores de $\delta^{13} \mathrm{C}$, en los carbonatos muestran valores contrastantes. Los carbonatos diseminados en La Ventilla y Cerro Gordo tienen valores bajos (- $6.7 \%$ y - $5.52 \%$, respectivamente), mientras que en San Pablo, es más alto (-2.83). En la materia orgánica de los paleosuelos asociados se documenta un valor de - $16.7 \%$ (Tabla 1 ).

\subsubsection{Suelos del Holoceno, fase II}

Aquí se incluyen a los suelos, pedosedimentos y sedimentos del último milenio. Comprenden suelos de escaso desarrollo con intercalaciones de arena y grava. Son Leptosoles o Fluvisoles primitivos. Las edades que se han registrado en estos materiales son diversas y contradictorias, ya que se presentan inversiones, tal que las edades más jóvenes se encuentran debajo de edades más antiguas (McClung et al., 2005). Los materiales orgánicos quemados han mostrado una mayor homogeneidad y congruencia, coincidiendo con la época colonial (Tabla 1).

\section{Discusión}

5.1. Distribución temporal de los paleosuelos de Teotihuacán y correlación con otros registros del centro de México:

\subsubsection{Paleosuelos del Pleistoceno, fase I}

Los paleosuelos que se incluyen en este periodo, entre 50000 y 14000 años A.P. se localizan, principalmente, en el fondo del valle de Teotihuacán. Se les ha descrito como SP3 y SP4 en la sección San Pablo (SolleiroRebolledo et al., 2011) y en Maseca como 2Btg y 3Btg (Solleiro-Rebolledo et al., 2006). En las partes elevadas, como en la ladera oriental de Cerro Gordo, el paleosuelo policíclico 2A, 2Bt, 3Bt, reportado por Solleiro-Rebolledo et al. (2006), es característico de este periodo. Todos ellos muestran horizontes arcillosos con coloraciones pardas y rasgos reductomórfico, y se han clasificado como Luvisoles crómicos y estágnicos (IUSS Working Group, 2006). En el transecto realizado (Figura 1), los paleosuelos formados en esta fase, se observan en el Cerro Gordo y en la Sierra Patlachique, en donde se presentan horizontes Bt sepultados. Es probable que también se encuentren en el fondo del valle, aunque sepultados a mayores profundidades, por la erosión intensa que ha afectado la región en diversos periodos. Los estudios realizados en San Pablo, por ejemplo, demuestran que estos paleosuelos se presentan a profundidades de más de $6 \mathrm{~m}$ (Solleiro-Rebolledo et al., 2011), en tanto que González et al., (2013) documenta su presencia en pedosedimentos (material de suelo incorporado en los sedimentos coluviales), localizados en el piedemonte de las sierras.
Es interesante que los perfiles de suelo en las partes elevadas (Cerro Gordo y Sierra Patlachique) no muestren carbonatos secundarios, diseminados en la matriz, o en forma de nódulos o concreciones. Sin embargo, entre las rocas volcánicas, se encuentran capas de caliches bien desarrollados, los cuales tienen edades de 35267 años (Tabla 1). Estos caliches, en otras posiciones geomorfológicas (partes bajas) están dentro de los paleosuelos. Consideramos que si bien los caliches están en sedimentos y rocas, su formación está relacionada con procesos pedogenéticos (aunque no se observen los paleosuelos), y que se correlacionan con los hallados en Maseca y San Pablo. Particularmente, en Maseca, se encuentran fracturas rellenas de carbonatos en los paleosuelos arcillosos, cuyas edades coinciden con las de Cerro Gordo. Probablemente, la erosión intensa que ha afectado al valle, sea responsable de la pérdida del registro edáfico.

Por otro lado, las firmas de isótopos estables de carbono son similares en ambos casos - 5.46\% (Cerro Gordo) y - 4.03 $\%$ (Maseca). De acuerdo con Cerling y Quade (1993), estos valores, en carbonatos, se relacionan con valores de $\delta^{13} \mathrm{C}$ en materia orgánica de -20.46 y - $19.03 \%$, respectivamente, lo que implica una contribución de una mezcla de plantas C3 y C4 (Lounejeva et al., 2006). De hecho, la firma más empobrecida en Cerro Gordo, que coincide con el valor del horizonte 2A, también de Cerro Gordo (Tabla 1), implica que hay una mayor proporción de plantas C3.

Con respecto a la distribución de esta unidad arcillosa en otras localidades dentro del valle de Teotihuacán, Hernández (2007) reporta edades de material carbonizado en varios paleosuelos limo-arcillosos hacia su porción oriente, que oscilan entre 30000 y 37000 años AP (no calibradas), que pueden ser correlacionados con esta fase.

En otros sitios del centro de México, el paleosuelo denominado fBo1 en Puebla-Tlaxcala (Heine, 1975), puede ser análogo, ya que su edad va de 21000 a 26000 años A.P. (no calibrada). Además, en la cuenca de México, se ha identificado el denominado Caliche I sobre horizontes arcillosos pardos (Bryan, 1948, Arellano 1953), presumiblemente con el mismo periodo de formación. Es interesante anotar que en los registros lacustres de Tecocomulco, Caballero et al. (1999) reportan la presencia de dos capas de caliches, una de las cuales se encuentra sobre un sedimento cuya edad es $30794 \pm 354$ años A.P.; la otra capa descansa sobre un sedimento de $19589 \pm 262$ años A.P. Por lo tanto, consideramos que estos carbonatos no son un caso único y aislado, sino que su distribución es constante en la cuenca de México.

\subsubsection{Paleosuelos del Pleistoceno, fase II}

Las edades que se tienen para caracterizar estos paleosuelos no son abundantes. En San Pablo, este periodo se ha caracterizado por una intensificación de los procesos geomórficos, que causaron una mayor incisión en el valle. De esta forma, el paleosuelo denominado SP2 (SolleiroRebolledo et al., 2011) es un Gleysol cortado por una serie 
de canales aluviales de orientación N-S. Asimismo, la presencia de gruesos paquetes de sedimentos encontrados en las barrancas, y que pueden corresponder con el tepetate basal, en el transecto estudiado (Figura 1), demuestra la inestabilidad ambiental. En la Cuenca de México, se ha asignado al sedimento Becerra una edad entre $11000 \mathrm{y}$ 16000 años, que incluye al Caliche II y a un paleosuelo denominado fBo2 cuya edad oscila entre 10000 y 12000 años (Heine, 1975), corroborando así la alta actividad geomórfica establecida para este periodo.

\subsubsection{Paleosuelos del Holoceno, fase I}

Durante el Holoceno temprano, en realidad son pocos los objetos paleopedológicos encontrados, ya que la mayor parte de las fechas publicadas (McClung de Tapia et al., 2005; Solleiro-Rebolledo et al., 2006, 2011; Sánchez et al., 2013) corresponden al Holoceno medio-tardío (Tabla 1). Esto se ha interpretado como un periodo de inestabilidad ambiental con mayor sedimentación que pedogénesis. De hecho, en diversos cortes se ha observado la presencia de gruesas capas de sedimentos volcánicos y coluvio-aluviales. Sin embargo, le existencia de un hiatus en las secuencias lacustres de la cuenca de México (Lozano-García y Ortega-Guerrero, 1998; Caballero et al., 2010) contradice esta hipótesis, ya que esta fase marcaría un periodo de no sedimentación y no depósito, misma que permitiría el desarrollo de suelos. Sedov et al. (2010) en Tepexpan, encuentran una secuencia de sedimentos lacustres desarrollada entre 10000 y 30000 años, en tanto que en los últimos 10000 años se tiene la formación de tres paleosuelos.

Por otro lado, el suelo Barrilaco (Bryan, 1948), formado entre 4500 y 7500 años A.P., contiene bandas de carbonatos pertenecientes al Caliche III cuya edad es de 5000 años A.P. (Heine y Ohngemach, 1976), que coincide con las edades en Teotihuacan, dentro del PNSP. Este paleosuelo representa este largo periodo de formación, e integra en un solo cuerpo, el resultado de varios ciclos de pedogenesis. Es por esta razón que consideramos que el PNSP cubre prácticamente todo el Holoceno.

El PNSP está bien desarrollado, es de color oscuro, con estructura en bloques angulares muy duros y consistentes. Existen rasgos particulares, que permiten identificar con mayor claridad el carácter poligenético. A nivel micromorfológico, en diferentes perfiles, se observa la presencia de cutanes de iluviación, afectados por los procesos de vertisolización, que los deforman, mostrando, entonces cutanes de estrés. Por otro lado, los carbonatos se ubican sobre todo en poros (Figura 8d) y sobre los cutanes de iluviación (Solleiro-Rebolledo et al., 2011). En el perfil 2 de la secuencia estudiada, se registra una estructura biogénica desarrollada sobre los bloques angulares, vérticos (Figura 8c) y la precipitación de carbonatos secundarios (Figura 8d). Estas características se interpretan como el resultado de una segunda fase de pedogénesis, la cual ocurre después de la formación de las propiedades vérticas, confirmando el carácter poligenético del paleosuelo. En el perfil 4, Canal, la iluviación de arcilla es, aún, más acentuada (Figura 8g). En general, en varios perfiles que muestran un enriquecimiento de arcilla, también se distinguen rasgos sedimentarios que indican redepositación de material. Es probable que esta redepositación sea uno de los mecanismos que influya en el contenido de arcilla. Al respecto, González et al. (2013) suponen que los Luvisoles pre-existentes, erosionados de las laderas y partes altas del valle de Teotihuacán, se encuentran como restos en el material parental del PNSP (Figura 8f).

\subsubsection{Suelos del Holoceno, fase II}

Estos materiales constituyen la cubierta edáfica actual. Los suelos de este periodo se caracterizan por su escaso grado de desarrollo, mostrando perfiles $\mathrm{A}, \mathrm{AC}$, en donde la sedimentación y la erosión han sido activos y limitan la pedogénesis. Es suelos se observan en las partes bajas del valle (perfiles 2, 3,4), en tanto que en las partes más elevadas, en donde la sedimentación no es tan intensa, los perfiles sí muestran el desarrollo de horizontes B.

\subsection{Distribución espacial de los paleosuelos}

Tomando como base los trabajos previos (SolleiroRebolledo et al., 2006, 2011; Sánchez et al., 2013), así como el transecto de la Figura 1, es claro que la distribución espacial de los paleosuelos de Teotihuacán es amplia. Sin embargo, gran parte de estos materiales está sepultado por sedimentos coluvio-aluviales. Por lo tanto, En la superficie se presentan suelos de escaso desarrollo clasificados como Fluvisoles. En las laderas, sin embargo, los paleosuelos maduros se encuentran muy cerca, o en la superficie. Esta situación hace que los Vertisoles poligenéticos (PNSP), que representan la fase de paleosuelos del Holoceno, fase I, se encuentren "soldados" con los Luvisoles (de la fase de paleosuelos del Pleistoceno I y II), formando pedocomplejos. Recientemente González et al. (2013) con base en investigaciones micromorfológicas concluyeron que los Luvisoles más antiguos contribuyeron con los materiales edafizados, arcillosos, al desarrollo de los Vertisoles más jóvenes. Las observaciones de la interrelación espacial de los Luvisoles pleistocénicos y Vertisoles holocénicos apoyan a la hipótesis de González et al. (2013) y confirman el posible vínculo evolucionista entre esos paleosuelos.

En el transecto estudiado (Figura 1), se ha reconstruido la distribución del PNSP, observándose que representa un continuo en las posiciones bajas del valle. La similitud en las características micromorfológicas (microestructura en bloques angulares, con cutanes de iluviación deformados y cutanes de estrés) confirma la identificación de esta unidad paleopedológica y apoya su correlación en varios perfiles. Sánchez et al. (2013) lo ubican PNSP, principalmente, en fondo del valle, entre altitudes de 2200 y $2400 \mathrm{msnm}$. Este paleosuelo puede ser considerado como un marcador estratigráfico, el cual, además, es de amplio interés, ya que en él ocurre la ocupación humana en el valle de Teotihuacán y el desarrollo de la agricultura. 
Tanto los suelos maduros más antiguos, como los Fluvisoles primitivos, se han combinado para formar un manto cuasicontinuo, debido a la alternancia de fases de estabilidad e inestabilidad geomorfológica. Durante los periodos de estabilidad, los paleosuelos se desarrollan, en tanto que en los periodos de inestabilidad, tienen lugar procesos de erosión y sedimentación, sepultando a los suelos previamente formados. Si los sedimentos tienen un espesor considerable (>1 m), separan claramente a los paleosuelos de la siguiente fase pedogenética. Pero cuando la sedimentación deja capas relativamente delgadas de materiales, éstos se integran al paleosuelo sepultado, y muestran, entonces contrastes marcado entre los horizontes. Por ejemplo, horizontes arcillosos subyaciendo a horizontes limosos (Figura 1).

5.3. Paleoambiente e impacto humano en la cubierta de suelos y paleosuelos

Las etapas de inestabilidad/estabilidad detectadas en los sedimentos y paleosuelos del valle de Teotihuacán obedecen a diversos procesos. Es probable que las diferencias, entre los paleosuelos del Pleistoceno, fases I y II, así como la fase inicial del Holoceno I, estén relacionadas a cambios naturales en el paisaje, ya sea climáticos o volcánicos/tectónicos. Al analizar el registro paleopedológico pleistocénico, se detectan ciertas contradicciones en el registro ambiental, ya que los Luvisoles se desarrollan en ambientes húmedos, en tanto que los carbonatos requieren ambientes más secos. De esta manera, la interpretación apunta a cambios climáticos entre la formación de los Luvisoles (que representan etapas húmedas) y la precipitación de los carbonatos (etapas secas). Desafortunadamente, el registro no permite determinar con precisión la duración de cada etapa, ya que los fechamientos obtenidos marcan momentos específicos de su formación. Por otro lado, se ha mencionado que las firmas de isótopos estables de carbono con valores que oscilan entre - 4.03 y - $7.68 \%$, los cuales se relacionan con valores de $\delta^{13} \mathrm{C}$ en materia orgánica de - 19.03 y - 22.68 \%o (Cerling y Quade, 1993). Estas variaciones en la composición revelan el cambio en la contribución de plantas $\mathrm{C} 4$ y C3, en diferentes momentos.

A diferencia, la etapa de inestabilidad, a finales del Holoceno I y durante el Holoceno II, parece estar más bien relacionada a los procesos antrópicos, los cuales modifican fuertemente la cubierta edáfica original (Rivera-Uria et al., 2007). Las prácticas agrícolas implementadas por los teotihuacanos, las cuales incluyeron formación de terrazas y canales de riego, entre otras, causaron un cambio en los rasgos pedogenéticos. Al respecto, Sánchez et al. (2013) comentan que la presencia del horizonte $\mathrm{AE}$ (de color claro) en el paleosuelo del Holoceno fase I, se debe a procesos de lavado causados por irrigación. Debido a que la localización del horizonte AE es exclusiva de las zonas más bajas, con pendientes casi planas, se le considera como una "anomalía ambiental" en la secuencia de horizontes del suelo negro teotihuacano. Es decir, se supone que su formación (y consecuente "blanqueamiento") se debe a la existencia de un volumen importante de agua de percolación, que propicia el lavado y la pérdida de materiales como los óxidos de hierro, arcillas e incluso ácidos húmicos. Este lavado no se relaciona a un aumento en la precipitación pluvial, ya que si lo fuera, tendría que encontrarse en diferentes posiciones del paisaje, o por lo menos en los sitios con pendientes de ligeras a moderadas.

De acuerdo a los datos generados, se supone que estos procesos están relacionados con la actividad agrícola del área. La irrigación de este suelo puede generar los procesos estágnicos observados, ya que el suelo se satura de agua y promueve la formación de los nódulos de hierro y el movimiento de los materiales (arcilla, materia orgánica), provocando eluviación (mostrada en el horizonte de coloración gris $\mathrm{AE}$ ) e iluviación en $\mathrm{AB}$ (con una mayor contenido de arcilla y materia orgánica). Sedov et al. (2009) reportan que el suelo negro contiene abundantes rasgos relacionados con el cultivo.

Por otro lado, durante la época colonial y reciente es notoria la intensificación de los procesos de erosión que han producido una cubierta edáfica heterogénea y suelos de pobre desarrollo.

5.4. Influencia de los elementos relictos (paleosuelos y caliches) en los procesos actuales de la ZC

Como se ha mencionado, la $\mathrm{ZC}$ representa el medio de interacción entre la roca, el agua, el aire, los organismos y el suelo, siendo este último el vínculo principal entre los diversos elementos (Lin, 2010). Considerando lo anterior, la cubierta edáfica moderna y los paleosuelos arcillosos con caliches forman parte de la ZC del valle de Teotihuacán.

Los carbonatos secundarios (tanto en caliches como diseminados) encontrados en zona de estudio se ubican en diversas geoformas, a profundidades diferentes (Figura 1). De acuerdo con las fechas obtenidas, es evidente que la formación de éstos responde a procesos multifásicos, que se originan, más comúnmente, en los paisajes del Pleistoceno. En el Holoceno, los carbonatos secundarios son más escasos, y los que se encuentran (Tabla 1), se presentan diseminados, o como recubrimientos sobre roca, es decir, no forman horizontes cementados. La mayor parte de las acumulaciones de carbonatos se concentran en los paleosuelos arcillosos (Luvisoles) (Tabla 1).

La importancia de los estos objetos paleopedológicos (paleosuelos y carbonatos) dentro de los estudios hidrológicos, es su lugar dentro de la ZC. En primer lugar, los paleosuelos arcillosos, tanto superficiales como sepultados, marcan una barrera o por lo menos limitan la percolación del agua. En segundo lugar, los horizontes de carbonatos representan una fuente para proveer de carbonatos a las aguas subterráneas. El hecho de que la mayoría de esos carbonatos se formaron en el Pleistoceno tardío, nos permite predecir que las características geoquímicas e isotópicas $\left({ }^{14} \mathrm{C}\right.$ y $\left.\delta^{13} \mathrm{C}\right)$, 
de una parte de los carbonatos disueltos en los acuíferos actuales, heredará una firma relicta y no va a corresponder a las condiciones y procesos ambientales actuales. Los experimentos de laboratorio han demostrado que el carbono inorgánico disuelto (CID) intercambia isótopos con los carbonatos sólidos (e.g. Wendt, 1971; Mozeto et al., 1984; Garnier, 1985). En muestras de acuíferos en calizas, se ha observado que existe un intercambio entre el CID y la matriz carbonatada (carbonatos sólidos). Si el contacto entre ambos elementos toma un tiempo suficientemente largo, el CID se aproximará al equilibrio isotópico con el carbonato sólido (Han et al., 2014). Por lo tanto, los valores isotópicos de ${ }^{14} \mathrm{C}$ como de $\delta^{13} \mathrm{C}$ del carbono disuelto en las aguas subterráneas se ven afectados por la composición de los carbonatos sólidos y su evolución geoquímica es similar (Han et al., 2014). Por otro lado, la contribución promedio del $\mathrm{CO}_{2}$ del suelo en el CID, en diferentes ríos en el mundo, se ha estimado en $67 \%$ (Berner et al., 1983; Ludwig et al., 1997), por lo que la firma isotópica resultante en el agua subterránea, refleja una contribución mixta, que no es sencilla de medir (Ludwig et al., 1997).

Es probable que los valores de isótopos estables de carbono encontrados en los carbonatos pedogéneticos del valle de Teotihuacán (oscilando entre - 4.03 y - $7.68 \%$ oo), así como el isótopo radioactivo $\left({ }^{14} \mathrm{C}\right)$ contribuyan con firmas particulares a las aguas subterráneas de la porción norte de la cuenca de México.

\section{Conclusiones}

La cubierta de suelos actual forma un patrón heterogéneo, en donde sobresalen los suelos de pobre desarrollo (Fluvisoles) formados en el último milenio. Sin embargo, dentro de este mosaico, también destaca la presencia de Vertisoles. Los estudios hechos en Teotihuacán han mostrado que este tipo de suelo se formó durante el Holoceno medio, y que representa la cubierta edáfica de la época teotihuacana. En muchos cortes (Figura 1) se presenta sepultado por Fluvisoles. En algunos otros, el sepultamiento es muy somero (Figura 10a), de manera que aparecen en superficie y se cultivan (Figura 10b). Además de los Vertisoles, se han encontrado paleosuelos arcillosos sepultados con carbonatos secundarios, que conforman una cubierta edáfica más antigua (pleistocénica). A pesar de que los procesos pedogenéticos han sufrido modificaciones, en función del cambio en los factores de formación, los paleosuelos se han integrado a los procesos modernos, repercutiendo en la complejidad de la distribución temporal y espacial de los materiales edáficos en el valle de Teotihuacán. Estos paleosuelos muestran propiedades diversas, que juegan un papel importante en los procesos de la zona crítica. Los paleosuelos más antiguos estudiados son arcillosos, de manera que pueden limitar los procesos de infiltración y recarga de los acuíferos. Asimismo, dentro de estos paleosuelos, formados en el Pleistoceno, encontramos capas de caliches, los cuales deben ser considerados porque pueden afectar las características geoquímicas e isotópicas del agua (valores de ${ }^{14} \mathrm{C}$ y $\delta^{13} \mathrm{C}$ ).

\section{Agradecimientos}

Este trabajo ha sido financiado por el proyecto CONACYT_166878 y PAPIIT_IN110710, así como el proyecto DFG-Alemania "Sekundärkarbonate in Böden arider Gebiete Mexikos als Indikatoren spätquartärer Umweltveränderungen". Agradecemos la colaboración de Jaime Díaz quien participó en las diferentes etapas del trabajo de campo, así como en la realización de las láminas delgadas.

\section{Referencias}

Arellano, R.V., 1953, Estratigrafía de la Cuenca de México: Memorias del Congreso Científico Mexicano, Comisión IV, 3, 172-186.

Barba, L., 2005, Materiales, técnicas y energía en la construcción, en RuizGallut, M.E., Torres-Peralta, J. (eds.), Arquitectura y urbanismo: a.

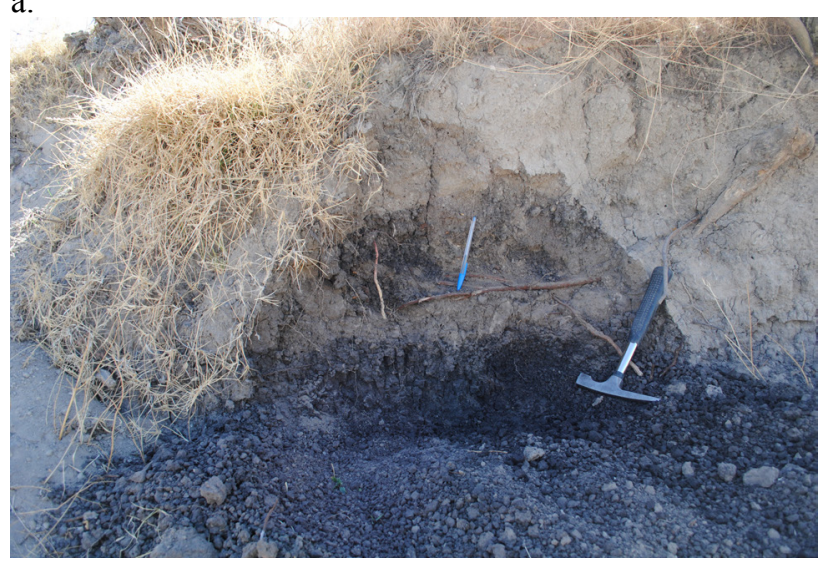

b.

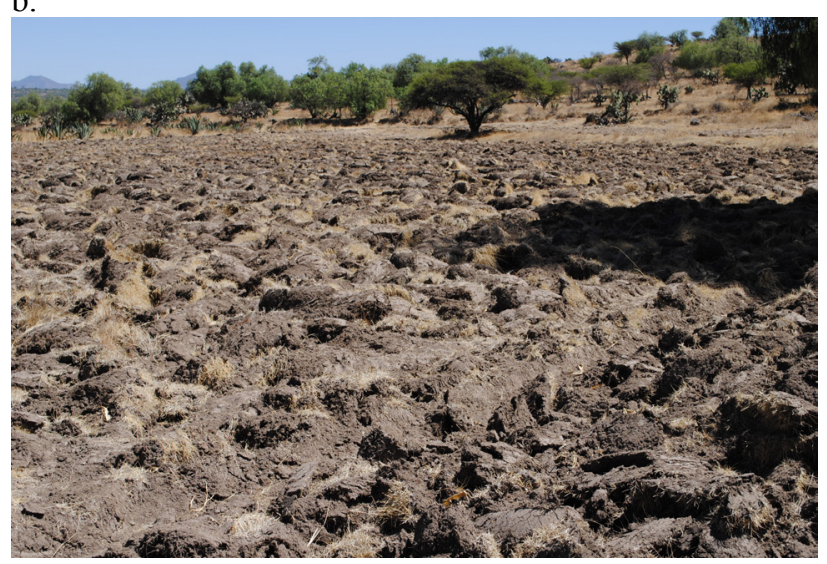

Figura 10. Vertisoles en el valle de Teotihuacán: a. sepultado por sedimentos coluviales; b. en superficie e incorporado a la agricultura actual. 
pasado y presente de los espacios en Teotihuacan: Memoria de la Tercera Mesa Redonda, Instituto Nacional de Antropología e Historia, 211-230 pp.

Berner, R.A., Lasaga, A.C., Garrels, R.M., 1983, The carbonate-silicate geochemical cycle and its effect on atmospheric carbon dioxide over the past 100 millions years: Am. J. Sci. 283, 641-683.

Bryan, K., 1948, Los suelos complejos y fósiles de la altiplanicie de México, en relación a los cambios climáticos: Boletín de la Sociedad Geológica Mexicana, 13, 1-20.

Cabadas, H., Solleiro-Rebolledo, E., Gama-Castro, J., Sedov, S., McClung, E., 2005, Paleosuelos como indicadores de cambio ambiental en el Cuaternario Superior. El caso del valle de Teotihuacan, en RuizGallut, M.E., Torres-Peralta J. (eds.), Arquitectura y urbanismo: pasado y presente de los espacios en Teotihuacan: Memoria de la Tercera Mesa Redonda, Instituto Nacional de Antropología e Historia, pp. 75-95.

Caballero, M., Lozano, G.S., Ortega, B., Urrutia, J., Macías, J.L., 1999, Environmental characteristics of lake Tecocomulco, northern basin of Mexico, for the last 50000 years: Journal of Paleolimnology, $22,399-411$.

Caballero, M., Lozano-García, S., Vázquez-Selem, L., Ortega, B., 2010, Evidencias de cambio climático y ambiental en registros glaciales y en cuencas lacustres del centro de México durante el último máximo glacial: Boletín de la Sociedad Geológica Mexicana, 62, 359-377.

Cabrera, R., 2005, Nuevas evidencias arqueológicas del manejo del agua en Teotihuacan. El campo y la ciudad, en Ruiz-Gallut, M.E., TorresPeralta, J. (eds.), Arquitectura y urbanismo: pasado y presente de los espacios en Teotihuacan: Memoria de la Tercera Mesa Redonda, Instituto Nacional de Antropología e Historia, 121-162, pp.

Carlos-Valerio, V., 2010, Procesos de Remoción en Masa en rocas volcánicas, porción SO de la cuenca de México: Posgrado en Ciencias de la Tierra, Universidad Nacional Autónoma de México, Tesis doctoral, $250 \mathrm{p}$.

Castilla, M.E., Tejero, J.D., 1987, Flora y Vegetación del Cerro Gordo (San Juan Teotihuacan) y regiones aledañas, Valle de México, México: Biótica, 12, 231-255.

Cerling, T.E., Quade, J., 1993, Stable carbon and oxygen isotopes in soil carbonates, en Swart, P.K., Lohmann, K.C., McKenzie, J.A., Savin, S.M. (Eds.), Climate Change in Continental Isotopic Records: American Geophysical Union Geophysical Monograph, 78, 217-231.

Flores-Román, D., Vela-Correa, G., Gama-Castro, J.E., Silva-Mora, L., 2009, Pedological diversity and the geoecological systems of Sierra de Guadalupe, central México: Revista Mexicana de Ciencias Geológicas, 26, 609-622.

Gama-Castro, J., Solleiro-Rebolledo, E., McClung, E., Villalpando, J.L., Sedov, S., Jasso-Castañeda, C., Palacios, S., Hernández, D., 2005, Contribuciones de la Ciencia del Suelo a la investigación arqueológicael caso de Teotihuacan: Revista Tierra Latinoamericana, 23, 1-11.

García, E., 1968, Clima actual de Teotihuacan, en Lorenzo, J.L. (Ed.). Materiales para la Arqueología de Teotihuacan XVII: INAH, México 10-27 pp.

García, E., 1988, Modificaciones al Sistema de Clasificación Climática de Köppen. México: Offset Larios, 219 p.

García-Palomo, A., Carlos-Valerio, V., López-Miguel, C., Galván-García, A., Concha-Dimas, A., 2006, Landslide inventory map of Guadalupe Range, north of the Mexico Basin: Boletín de la Sociedad Geológica Mexicana, LVIII, 195-204.

Garnier, J.-M., 1985, Retardation of dissolved radiocarbon through a carbonated matrix: Geochimica et Cosmochimica Acta, 49, 683-693.

González, S., Jiménez-López, J.C., Hedges, R., Huddart, D., Ohman, J.C., Turner, R., Pompa y Padilla, J.A., 2003, Earliest humans in the Americas: new evidence from Mexico: Journal of Human Evolution, 44, 379-387.

González, S., Jiménez-López, J.C., Hedges, R., Pompa y Padilla, J.A., Huddart, D., 2006, Early Humans in Mexico: New Chronological Data, en Jiménez-López, J.C., González, S., Pompa y Padilla, J.A., Ortiz-Pedraza, F. (eds.), El Hombre Temprano en América y sus implicaciones en el poblamiento de la Cuenca de México: Primer Simposio Internacional, Instituto Nacional de Antropología e
Historia, México, DF, 67-76 pp.

González, M.L., Vázquez-Selem L., Gama Castro J.E., McClung de Tapia E., Sedov S., 2013, History of pedogenesis and geomorphic processes in the Valley of Teotihuacán, Mexico: Micromorphological evidence from a soil catena: Spanish Journal of Soil Science, 3, 201-216.

Han, L.F., Plummer, L.N., Aggarwal, P., 2014, The curved ${ }^{14} \mathrm{C}$ vs. $\delta^{13} \mathrm{C}$ relationship in dissolved inorganic carbon: A useful tool for groundwater age- and geochemical interpretations: Chemical Geology, 387, 111-125.

Heine, K., 1975, Studien zur jungquartären Glazialmorphologie mexikanischer Vulkane mit einem Ausblick auf die Klimaentwicklung: Franz Steiner Verlag GMBH, Wiesbaden, Germany, 178 p.

Heine, K., Ohngemach, D., 1976, Die Pleistozän/Holozän-Grenze in Mexiko: Münster. Forschung Geol. Paläont., 38-39, 229-251.

Hernández, J.I., 2007, Geología y geomorfología de la región de los yacimientos de obsidiana de Otumba en el sector norte de la Sierra Nevada de México: Facultad de Filosofía y Letras, Universidad Nacional Autónoma de México, tesis profesional, $125 \mathrm{p}$.

IUSS Working Group WRB, 2006, World Reference Base for Soil Resources 2006. A Framework for International Classification, Correlation and Communication. International Union of Soil Science. Food and Agriculture Organization of the United Nations, Rome. 104 pp.

Lin, H., 2010, Earth's Critical Zone and hydropedology: concepts, characteristics, and advances: Hydrology and Earth System Sciences, $14,25-45$.

Lin, H., Bouma, J., Pachepsky, Y., Western, A., Thompson, J., 2006, Hydropedology: Synergistic integration of pedology and hydrology: Water Resources Research, 42, W05301, 1-13.

López-Austin A. 1989, La historia de Teotihuacan, en López-Austin, A., Romero-Galván, J.R, Martínez, C. (eds,), Teotihuacan: El Equilibrista-Turner libros México-Madrid, pp. 13-35.

Lounejeva, E., Morales-Puente, P., Cabadas-Báez, H., CienfuegosAlvarado, E., Sedov, S., Vallejo-Gómez, E., Solleiro-Rebolledo, E., 2006, Late Pleistocene to Holocene environmental changes from $\delta^{13} \mathrm{C}$ determinations in soils at Teotihuacan, Mexico: Geofísica Internacional, 45, 85-98.

Lozano-García, S., Ortega-Guerrero, B., 1998, Late Quaternary environmental changes of the central part of the Basin of Mexico; correlation between Texcoco and Chalco Basins: Review of Paleobotany and Palinology, 99, 77-93.

Ludwig, W., Amiotte Suchet, P., Munhoven, G., Probst, J.L., 1997, Atmospheric $\mathrm{CO}^{2}$ consumption by continental erosion: present-day control and implications for the last glacial maximum: Global and Planetary Change, 16-17, 107-120.

Manzanilla, L., 1997, The Impact of Past Climate Change on Past Civilizations: A Revisionist Agenda for Future Research: Quaternary International, 43-44, 153-159.

McClung de Tapia, E., 1987, Agriculture and the Formation of the Teotihuacan State, en Manzanilla, L. (ed.), Studies in the Neolithic and Urban Revolutions: The V. Gordon Childe Colloquium, Mexico, 1986: BAR International Series 349, Oxford, England, 353-364 pp.

McClung de Tapia, E., 2012, Silent Hazards, Invisible Risks: Prehispanic Erosion in the Teotihuacan Valley, Central Mexico, en Cooper, J., Sheets, P., Surviving Sudden environmental Change, Understanding Hazards, Mitigating impacts, avoiding disasters: University press of Colorado, 143-165 pp.

McClung de Tapia, E., Solleiro-Rebolledo, E., Gama Castro, J., Villalpando, J.L., Sedov, S., 2003, Paleosols in the Teotihuacan valley, Mexico: evidence for paleoenvironment and human impact: Revista Mexicana de Ciencias Geológicas, 20, 270-282.

McClung de Tapia, E., Domínguez, I., Gama, J., Solleiro-Rebolledo, E., Sedov, S., 2005, Radiocarbon dates from soil profiles in the Teotihuacan valley, Mexico: geomorphological processes and vegetation change: Radiocarbon, 47, 159-175.

Millon, R., 1973, Urbanization at Teotihuacan, Mexico, vol. 1: The Teotihuacan Map. University of Texas Press, Austin, 154 p.

Mooser, F., 1975, Historia geológica de la Cuenca de México: Memorias de las Obras del Sistema de Drenaje Profundo del Distrito Federal, 
DDF, Tomo I, 7-38.

Mozeto, A.A., Fritz, P., Qureshi, R.M., 1984, Laboratory study on carbon isotope uptake by calcite from carbonate in aqueous solution: Isotope Hydrology, 1983IAEA, 591-602.

National Research Council, 2001, Basic research opportunities in earth science: National Research Council, National Academy Press, Washington DC, USA, 154 p.

Rivera-Uria, M.Y., Sedov, S., Solleiro-Rebolledo, E., Pérez-Pérez, J., McCLung, E., González, A., Gama-Castro, J., 2007, Degradación ambiental en el valle de Teotihuacan: evidencias geológicas y paleopedológicas: Boletín de la Sociedad Geológica Mexicana, 59 (2), 203-217.

Rzedowski, J., Guzmán, G., Hernández, A., Muñiz, C., 1964, Cartografía de la vegetación de la parte norte del valle de México: México, D.F. Anales de la Escuela Nacional de Ciencias Biológicas, Instituto Politécnico Nacional, 13, 31-57.

Sánchez, S., Solleiro-Rebolledo, E., Sedov, S., McClung de Tapia, E., Golyeva, A., Prado, B., Ibarra-Morales, E., 2013, The Black San Pablo paleosol of the Teotihuacan Valley, Mexico: pedogenesis, fertility, and use in ancient agricultural and urban systems: Geoarchaeology, 28, 249-267.

Sanders, W.T., Parsons, J.R. Santley, R.S., 1979, The Basin of Mexico: Ecological Processes in the Evolution of a Civilization. Academic Press, New York, 575 p.

Sedov, S., Rivera, Y., Solleiro-Rebolledo, E., 2009, Holocene paleoecology of Teotihuacan, Mexico: micromorphological evidences from alluvial paleosols and pedosediments: Frankfurter Geowissenschaftliche Arbeiten Serie D Physische Geographie, Band 30, 123-131.

Sedov, S., Lozano-García, S., Solleiro-Rebolledo, E., McClung de Tapia, E., Ortega-Guerrero, B., Sosa-Nájera, S., 2010, Tepexpan revisited: a multiple proxy of local environmental changes in relation to human occupation from a lake shore section in Central Mexico: Geomorphology, 122, 309-322.

Solleiro-Rebolledo, E., Sedov, S., McClung E., Cabadas H., Gama-Castro J., Vallejo-Gómez E., 2006, Spatial variability of environment change in the Teotihuacan valley during late Quaternary: paleopedological inferences. Quaternary International, 156-157, 13-31.

Solleiro-Rebolledo, E., Sycheva, S., Sedov, S., McClung de Tapia, E., Rivera-Uria, Y., Salcido-Berkovich, C., Kuznetsova, A., 2011, Fluvial processes and paleopedogenesis in the Teotihuacan Valley, México: Responses to late Quaternary environmental changes: Quaternary International 233, 40-52.

Sycheva, S.A., Sedov S.N., Solleiro-Rebolledo, E., 2010, Izmeneniya landshaftov dolini r. San-Pablo v konce pozdnego pleistocena (Teotihuacan, Mexico) (Late Pleistocene landscape changes in the San Pablo valley (Teotihuacan, Mexico): Geomorfologia, 3, 90-100.

Tapia-Varela, G., López-Blanco, J., 2002, Mapeo geomorfológico analítico de la porción central de la Cuenca de México: unidades morfogenéticas a escala 1:100000: Revista Mexicana de Ciencias Geológicas, 19, 50-65.

Vázquez, E.R., Jaimes, P., 1989, Geología de la Cuenca de México: Geofísica Internacional, 28, 133-189.

Wendt, I., 1971, Carbon and oxygen isotope exchange between $\mathrm{HCO}_{3}$ in saline solutions and $\mathrm{CaCO}_{3}$ : Earth Planet. Sci. Lett., 12, 439-442.

Manuscrito recibido: Marzo 4, 2014

Manuscrito corregido recibido: Noviembre 13, 2014

Manuscrito aceptado: Noviembre 24, 2014 